\title{
Fibred GK geometry and supersymmetric AdS solutions
}

\author{
Jerome P. Gauntlett, ${ }^{a}$ Dario Martelli ${ }^{b, c}$ and James Sparks ${ }^{d}$ \\ ${ }^{a}$ Blackett Laboratory, Imperial College, \\ Prince Consort Rd., London SW7 2AZ, U.K. \\ ${ }^{b}$ Dipartimento di Matematica "Giuseppe Peano", Università di Torino, \\ Via Carlo Alberto 10, Torino 10123, Italy \\ ${ }^{c}$ INFN, Sezione di Torino and Arnold-Regge Center, \\ Via Pietro Giuria 1, Torino 10125, Italy \\ ${ }^{d}$ Mathematical Institute, University of Oxford, Andrew Wiles Building, \\ Radcliffe Observatory Quarter, Woodstock Road, Oxford OX2 6GG, U.K. \\ E-mail: j.gauntlett@imperial.ac.uk, dario.martelli@unito.it, \\ james.sparks@maths.ox.ac.uk
}

ABSTRACT: We continue our study of a general class of $\mathcal{N}=2$ supersymmetric $A d S_{3} \times Y_{7}$ and $A d S_{2} \times Y_{9}$ solutions of type IIB and $D=11$ supergravity, respectively. The geometry of the internal spaces is part of a general family of "GK geometries", $Y_{2 n+1}, n \geq 3$, and here we study examples in which $Y_{2 n+1}$ fibres over a Kähler base manifold $B_{2 k}$, with toric fibres. We show that the flux quantization conditions, and an action function that determines the supersymmetric $R$-symmetry Killing vector of a geometry, may all be written in terms of the "master volume" of the fibre, together with certain global data associated with the Kähler base. In particular, this allows one to compute the central charge and entropy of the holographically dual $(0,2)$ SCFT and dual superconformal quantum mechanics, respectively, without knowing the explicit form of the $Y_{7}$ or $Y_{9}$ geometry. We illustrate with a number of examples, finding agreement with explicit supergravity solutions in cases where these are known, and we also obtain new results. In addition we present, en passant, new formulae for calculating the volumes of Sasaki-Einstein manifolds.

KeYwords: AdS-CFT Correspondence, Differential and Algebraic Geometry, Black Holes in String Theory

ArXIV EPRINT: 1910.08078 


\section{Contents}

1 Introduction $\quad 2$

2 GK geometry and the extremal problem 3

3 Geometry of the toric $X_{2 r+1}$ fibre $\quad 6$

3.1 Toric Kähler cones $\quad 7$

$\begin{array}{lll}3.2 & \text { The master volume } & 8\end{array}$

4 Fibred GK geometry 11

4.1 General set-up 11

$4.2 \quad X_{2 r+1} \hookrightarrow Y_{2 r+3} \rightarrow B_{2} \quad 14$

$4.3 X_{2 r+1} \hookrightarrow Y_{2 r+5} \rightarrow B_{4} \quad 15$

$4.4 X_{2 r+1} \hookrightarrow Y_{2 r+7} \rightarrow B_{6} \quad 16$

5 Examples $\quad 17$

$\begin{array}{ll}5.1 \text { Type IIB } & 17\end{array}$

$\begin{array}{lll}5.1 .1 & B_{4}=d P_{k} & 18\end{array}$

$\begin{array}{lll}5.1 .2 & B_{4}=\Sigma_{g_{1}} \times \Sigma_{g_{2}} & 19\end{array}$

$\begin{array}{lll}5.1 .3 \quad B_{4}=\mathbb{F}_{n} & 20\end{array}$

$\begin{array}{lll}5.2 D=11 & 22\end{array}$

$\begin{array}{lll}6 & \text { Discussion } & 24\end{array}$

$\begin{array}{ll}\text { A Master volume identities } & 24\end{array}$

$\begin{array}{lll}\text { B } & \text { Master volume for } X_{3} & 27\end{array}$

C More on the master volume $\quad 28$

$\begin{array}{lll}\text { C.1 Derivatives of the master volume } & 28\end{array}$

C.2 Formulae for $X_{2 r+1} \hookrightarrow Y_{2 r+5} \rightarrow B_{4}$

D Explicit solutions with Kähler-Einstein factors $\quad 34$

D.1 Type IIB: $\mathscr{Y Y}^{\mathrm{p}, \mathrm{q}}\left(\mathrm{KE}_{4}^{+}\right) \quad 34$

D.2 $D=11: \mathscr{Y}^{\mathrm{p}, \mathrm{q}}\left(\mathrm{KE}_{6}^{+}\right)$

$\begin{array}{ll}\text { E Sasakian volume function } & 38\end{array}$ 


\section{Introduction}

An interesting arena for exploring the AdS/CFT correspondence, both from the geometric and the field theory points of view, is the class of supersymmetric $A d S_{3} \times Y_{7}$ solutions of type IIB supergravity of [1] and $A d S_{2} \times Y_{9}$ solutions of $D=11$ supergravity of [2]. These solutions are dual to $d=2$ SCFTs preserving $(0,2)$ supersymmetry and an superconformal quantum mechanics preserving $\mathcal{N}=2$ supersymmetry, respectively, both of which have an abelian $R$-symmetry. The internal spaces of these supergravity solutions are lowdimensional examples of a novel kind of geometry, called "GK geometry", which is defined on odd-dimensional manifolds $Y_{2 n+1}, n \geq 3$ [3]. GK geometry consists of a Riemannian metric, a scalar function $B$ and a closed two-form $F$ which extremizes a particular action and also admits a certain type of Killing spinor. Furthermore, motivated by the supergravity solutions, there is a natural flux quantization condition that can be imposed on cycles of co-dimension two. The GK geometries have a canonical $R$-symmetry Killing vector which, for the supergravity solutions, is precisely dual to the $R$-symmetry in the dual field theory.

It has been shown recently that the $R$-symmetry Killing vector in GK geometry can be obtained via an interesting variational problem [4] that is analogous to the principle of volume minimization in Sasaki-Einstein geometry $[5,6]$. In the case of $n=3$, i.e. $Y_{7}$, this variational problem is a geometric realisation of the $c$-extremization principle for the dual $(0,2) d=2$ SCFTs proposed in [7] and allows one to obtain, for example, the central charge of the dual field theory without knowing the explicit $A d S_{3} \times Y_{7}$ solution. For the case of $n=4$, i.e. $Y_{9}$, there is, in general, no analogous extremization principle in field theory that one can compare with. However, for special subclasses of $Y_{9}$ it corresponds to the $I$-extremization principle of [8], as shown in [9, 10]. Furthermore, when the $A d S_{2} \times Y_{9}$ solution arises as the near horizon limit of a black hole, the geometric variational problem also allows one to calculate the entropy of the black hole, again without knowing the explicit supergravity solution [4]. In particular, for the class of such black hole solutions that asymptotically approach $A d S_{4}$, the connection with $I$-extremization provides a microscopic derivation of the black hole entropy, substantially extending [8] (for other related work see, for example, [11-15]).

In previous work $[9,16]$, the variational problem of [4] was utilised to study specific classes of $Y_{7}$ and $Y_{9}$ that arise as a fibration over a Riemann surface $B_{2}=\Sigma_{g}$ with toric fibres $X_{5}$ and $X_{7}$, respectively. By taking the $R$-symmetry Killing vector field to be tangent to the fibres it was shown that the general formulae in [4] can be recast in terms of a master volume formula for the toric fibres which is a function of the toric data, a choice of $R$-symmetry vector and an arbitrary transverse Kähler class. It was shown that the extremization problem can be implemented using the master volume formula combined with a set of integers that determine the fibration of $X_{5}$ or $X_{7}$ over $\Sigma_{g}$, as well as a Kähler class parameter for $\Sigma_{g}$.

In this paper we substantially generalize these results. We will study the extremal problem for GK geometry on $Y_{2 n+1}$ that arise as fibrations of the form $X_{2 r+1} \hookrightarrow Y_{2 r+2 k+1} \rightarrow$ $B_{2 k}$, with $r \geq 1, k \geq 1$ and $r+k=n \geq 3$. We will assume that the base manifold $B_{2 k}$ of the fibration is Kähler, while the fibre is again taken to be toric. Remarkably, we will 
show that the extremal problem of [4] can again be implemented using the master volume formula for the toric fibres, as in the cases studied in $[9,16]$. One new feature is that while for $k=1$ derivatives of the master volume with respect to the $R$-symmetry vector and Kähler class parameters appear, for $k>1$ we will also need to consider derivatives with respect to the toric data. We will present explicit formulae for specific values of $r, k$ that are associated with interesting $A d S_{3}$ and $A d S_{2}$ solutions, but it is reasonably clear how to extend to other values. A simple explicit expression for the master volume formula in terms of the toric data was given for $X_{5}$ and $X_{7}$ in [9, 16], respectively. Here we will also provide an analogous expression for the simpler case of $X_{3}$.

For application to the AdS/CFT correspondence the main utility of our new results is that one can calculate quantities of physical interest without knowing the explicit supergravity solutions, just assuming that they exist. That being said, it is very satisfying to be able to check the new formulae that we derive here against some explicitly known solutions. We will carry out such checks for the class of $A d S_{3} \times Y_{7}$ solutions of type IIB found in [17] with $X_{3} \hookrightarrow Y_{7} \rightarrow B_{4}$, i.e. $r=1, k=2$, with $B_{4}$ having a Kähler-Einstein metric. We will also carry out a similar check for a class of $A d S_{2} \times Y_{9}$ solutions of $D=11$ supergravity with $X_{3} \hookrightarrow Y_{9} \rightarrow B_{6}$, i.e. $r=1, k=3$, with $B_{6}$ having a Kähler-Einstein metric. These latter solutions were constructed in [18] and here we complete the analysis of flux quantization.

The plan of the rest of the paper is as follows. In section 2 we summarize general aspects of GK geometry and the associated extremal problem. In section 3 we discuss the toric fibres and their master volume. In section 4 , which contains our main new results, we present the formulae for implementing the extremal problem in the fibred GK geometries for Kähler base manifolds of dimension $k=1,2,3$. We illustrate the formulae considering a variety of examples in section 5 , focusing on the new cases of $k=2$ and $k=3$. In addition to reproducing the results of some known explicit supergravity solutions, where the bases $B_{4}$ and $B_{6}$ are Kähler-Einstein manifolds, we also work out examples where the base manifold is Kähler, but not Einstein. In particular, we consider $B_{4}=\Sigma_{g_{1}} \times \Sigma_{g_{2}}$, the product of two Riemann surfaces of genus $g_{1}$ and $g_{2}$, as well as $B_{4}=\mathbb{F}_{n}$, the $n$-th Hirzebruch surface. We conclude in section 6 with some discussion. The appendices A-D contain the derivations of the various key identities involving the master volume, that we use in the main part of the paper. We have also included an appendix E, which explains how the formalism developed in $[9,16]$ and the present paper allows one to efficiently compute the Sasakian volume function of $[5,6]$.

\section{GK geometry and the extremal problem}

We begin by briefly summarizing some aspects of GK geometry [3]. This is a geometry defined on an odd-dimensional manifold, $Y_{2 n+1}$, with $n \geq 3$, consisting of a metric, a scalar function $B$ and a closed two-form $F$, so that $\mathrm{d} F=0$.

The existence of "supersymmetry", by which we mean the existence of certain Killing spinors given in [3], implies that the metric on $Y_{2 n+1}$ has a unit norm Killing vector $\xi$, called the $R$-symmetry vector field. Since $\xi$ is nowhere vanishing it defines a foliation $\mathcal{F}_{\xi}$ 
of $Y_{2 n+1}$. In local coordinates we may write

$$
\xi=\frac{1}{c} \partial_{z}, \quad \eta=c(\mathrm{~d} z+P),
$$

where $c \equiv(n-2) / 2$ and $\eta$ is the Killing one-form dual to $\xi$. The metric on $Y_{2 n+1}$ then has the form

$$
\mathrm{d} s_{2 n+1}^{2}=\eta^{2}+\mathrm{e}^{B} \mathrm{~d} s_{2 n}^{2},
$$

where $\mathrm{d} s_{2 n}^{2}$ is a Kähler metric transverse to $\mathcal{F}_{\xi}$. This Kähler metric, with transverse Kähler two-form $J$, Ricci two-form $\rho=\mathrm{d} P$ and Ricci scalar $R$, determines all of the remaining fields. Specifically,

$$
\mathrm{e}^{B}=\frac{c^{2}}{2} R, \quad F=-\frac{1}{c} J+\mathrm{d}\left(\mathrm{e}^{-B} \eta\right) .
$$

In particular, notice that we require positive scalar curvature, $R>0$. These off-shell "supersymmetric geometries" become on-shell GK geometries, or "supersymmetric solutions", provided that the transverse Kähler metric satisfies the non-linear partial differential equation

$$
\square R=\frac{1}{2} R^{2}-R_{i j} R^{i j}
$$

For $n=3$ and $n=4$ these give rise to supersymmetric $A d S_{3} \times Y_{7}$ and $A d S_{2} \times Y_{9}$ solutions of type IIB and $D=11$ supergravity, which we describe in more detail below. For these cases we must impose a flux quantization condition for cycles of codimension two, and this naturally generalizes to all $n \geq 3$. Specifically, if $\Sigma_{A}$ are a basis for the free part of $H_{2 n-1}\left(Y_{2 n+1}, \mathbb{Z}\right)$ we impose

$$
\int_{\Sigma_{A}}\left[\eta \wedge \rho \wedge \frac{1}{(n-2) !} J^{n-2}+\frac{c}{2} *_{2 n} \mathrm{~d} R\right]=\nu_{n} N_{A}
$$

where $N_{A} \in \mathbb{Z}$ and the non-zero, real constant $\nu_{n}$ is explicitly fixed only for the cases of $n=3$ and $n=4$, as given below.

We also recall that for an off-shell supersymmetric geometry (i.e. not imposing (2.4)) the real cone over $Y_{2 n+1}, C\left(Y_{2 n+1}\right) \equiv \mathbb{R}_{>0} \times Y_{2 n+1}$, equipped with the conical metric

$$
\mathrm{d} s_{2 n+2}^{2}=\mathrm{d} \varrho^{2}+\varrho^{2} \mathrm{~d} s_{2 n+1}^{2},
$$

has some important properties. The cone $C\left(Y_{2 n+1}\right)$ has an integrable complex structure, and there exists a nowhere vanishing holomorphic $(n+1,0)$-form $\Psi$, which, furthermore, is closed $\mathrm{d} \Psi=0$. It follows that $C\left(Y_{2 n+1}\right)$ has vanishing first Chern class. Additionally, the $R$-symmetry vector $\xi$ is holomorphic, and moreover $\Psi$ has a fixed charge with respect to the $R$-symmetry vector:

$$
\mathcal{L}_{\xi} \Psi=\frac{\mathrm{i}}{c} \Psi
$$


We can now summarize the extremal problem for the off-shell supersymmetric geometry that was presented in [4]. We fix a complex cone $C\left(Y_{2 n+1}\right)=\mathbb{R}_{>0} \times Y_{2 n+1}$ with holomorphic volume form $\Psi$, and holomorphic $\mathrm{U}(1)^{s}$ action. We then choose a fiducial holomorphic $R$-symmetry vector $\xi$ and demand that the holomorphic volume form has fixed charge $1 / c$, as in (2.7). This choice of $\xi$ defines a foliation $\mathcal{F}_{\xi}$, and we then further choose a transverse Kähler metric with basic cohomology class $[J] \in H_{B}^{1,1}\left(\mathcal{F}_{\xi}\right)$. We do not impose the condition (2.4), as this would immediately put us on-shell. However, in order to impose the flux quantization condition (2.5) we impose that the integral of (2.4) is satisfied. Specifically, we impose the topological constraint

$$
\int_{Y_{2 n+1}} \eta \wedge \rho^{2} \wedge \frac{1}{(n-2) !} J^{n-2}=0
$$

and also impose the flux quantization conditions ${ }^{1}$

$$
\int_{\Sigma_{A}} \eta \wedge \rho \wedge \frac{1}{(n-2) !} J^{n-2}=\nu_{n} N_{A}
$$

with the basis of cycles $\left\{\Sigma_{A}\right\}$ all tangent to $\xi$. Finally, an on-shell geometry, with properly quantized flux, extremizes the supersymmetric action

$$
S_{\mathrm{SUSY}}=\int_{Y_{2 n+1}} \eta \wedge \rho \wedge \frac{1}{(n-1) !} J^{n-1} .
$$

For a given $\xi$, it is important to emphasize that the quantities (2.8), (2.9) and (2.10) just depend on the basic cohomology class $[J] \in H_{B}^{1,1}\left(\mathcal{F}_{\xi}\right)$, and not on $J$ itself [4]. Thus, for fixed $[J]$, we are extremizing over the space of $R$-symmetry vectors. A GK geometry with quantized flux is necessarily an extremal point, although as discussed in $[4,16]$ for a given extremal point there may be obstructions to the existence of a corresponding GK supergravity solution, satisfying (2.4).

For the case of $n=3$, i.e. $Y_{7}$, the above extremal problem is associated to supersymmetric solutions of type IIB supergravity of the form

$$
\begin{aligned}
\mathrm{d} s_{10}^{2} & =L^{2} \mathrm{e}^{-B / 2}\left(\mathrm{~d} s^{2}\left(A d S_{3}\right)+\mathrm{d} s^{2}\left(Y_{7}\right)\right), \\
F_{5} & =-L^{4}\left(\operatorname{vol}_{A d S_{3}} \wedge F+*_{7} F\right),
\end{aligned}
$$

where $\mathrm{d} s^{2}\left(A d S_{3}\right)$ has unit radius, and $L>0$ is a constant. The five-form $F_{5}$ is properly quantized provided that we choose the constant $\nu_{3}$ to be

$$
\nu_{3}=\frac{2\left(2 \pi \ell_{s}\right)^{4} g_{s}}{L^{4}}
$$

where $\ell_{s}$ is the string length, and $g_{s}$ is the constant string coupling. Furthermore, the value of the on-shell action also determines the central charge, $c_{\text {sugra }}$, of the dual field theory. Specifically, defining the "trial central charge", $\mathscr{Z}$, via

$$
\mathscr{Z} \equiv \frac{3 L^{8}}{(2 \pi)^{6} g_{s}^{2} \ell_{s}^{8}} S_{\mathrm{SUSY}}=\frac{12(2 \pi)^{2}}{\nu_{3}^{2}} S_{\mathrm{SUSY}}
$$

\footnotetext{
${ }^{1}$ As discussed in [4], this is equivalent to the flux quantization condition if $H^{2}\left(Y_{2 n+1}, \mathbb{R}\right) \cong H_{B}^{2}\left(\mathcal{F}_{\xi}\right) /[\rho]$, which holds in the classes of examples studied in this paper.
} 
where $S_{\text {SUSY }}$ is the supersymmetric action (2.10) with $n=3$, then we have

$$
\left.\mathscr{Z}\right|_{\text {on-shell }}=c_{\text {sugra }} \text {. }
$$

Similarly, when $n=4$, i.e. $Y_{9}$, the above extremal problem is associated to supersymmetric solutions of $D=11$ supergravity of the form

$$
\begin{aligned}
\mathrm{d} s_{11}^{2} & =L^{2} \mathrm{e}^{-2 B / 3}\left(\mathrm{~d} s^{2}\left(A d S_{2}\right)+\mathrm{d} s^{2}\left(Y_{9}\right)\right), \\
G_{4} & =L^{3} \operatorname{vol}_{A d S_{2}} \wedge F,
\end{aligned}
$$

where $\mathrm{d} s^{2}\left(A d S_{2}\right)$ has unit radius. The four-form $G_{4}$ (or more precisely the Hodge dual seven-form $\left.*_{11} G_{4}\right)$ is properly quantized provided that we choose the constant $\nu_{4}$ to be

$$
\nu_{4}=\frac{\left(2 \pi \ell_{p}\right)^{6}}{L^{6}}
$$

where $\ell_{p}$ is the eleven-dimensional Planck length. For this case we can define a "trial entropy", $\mathscr{S}$, via

$$
\mathscr{S} \equiv \frac{4 \pi L^{9}}{(2 \pi)^{8} \ell_{p}^{9}} S_{\mathrm{SUSY}}
$$

where $S_{\text {SUSY }}$ is the supersymmetric action $(2.10)$ with $n=4$. In the case that the $D=11$ solution arises as the near-horizon limit of a supersymmetric black hole, it is expected that $\left.\mathscr{S}\right|_{\text {on-shell }}$ is the entropy of the black hole [4]. More generally, it is expected that $\left.\mathscr{S}\right|_{\text {on-shell }}$ is the logarithm of a supersymmetric partition function of the dual quantum mechanical theory [4]. For the sub-class of solutions for which $Y_{9}$ is the total space of a fibration of $X_{7}$ over a Riemann surface there is also an established connection with $\mathcal{I}$-extremization $[9,10]$, which provides a state counting interpretation of the entropy of infinite classes of supersymmetric, asymptotically $A d S_{4}$ black hole solutions.

In the remainder of the paper we will be interested in implementing the above extremal problem for geometries in which $Y_{2 n+1}$ takes the fibred form

$$
X_{2 r+1} \hookrightarrow Y_{2 r+2 k+1} \rightarrow B_{2 k},
$$

where $n=r+k$ and $B_{2 k}$ is a Kähler manifold. We will further restrict to the case that the fibre manifold $X_{2 r+1}$ is toric, so that the cone metric over $X_{2 r+1}$ is invariant under a holomorphic $\mathrm{U}(1)^{r+1}$ isometry, and moreover we take the $R$-symmetry vector $\xi$ to be tangent to the toric fibre. We describe this geometry in more detail in the next section.

\section{Geometry of the toric $X_{2 r+1}$ fibre}

In this section we describe the geometry of the fibres $X_{2 r+1}$ in (2.18), in particular introducing the so-called master volume $\mathcal{V}$. Our discussion here summarizes and generalizes section 3 of [16] from dimension $r=2$ to arbitrary dimension, and in addition we derive some new identities satisfied by the master volume that will be important later in the paper. 


\subsection{Toric Kähler cones}

We start by assuming that we have a toric Kähler cone, $C\left(X_{2 r+1}\right)$, in real dimension $2(r+1)$. By definition these are Kähler metrics of the conical form

$$
\mathrm{d} s_{C\left(X_{2 r+1}\right)}^{2}=\mathrm{d} \varrho^{2}+\varrho^{2} \mathrm{~d} s_{2 r+1}^{2},
$$

that admit a $\mathrm{U}(1)^{r+1}$ action. This action is taken to be generated by the holomorphic Killing vectors $\partial_{\varphi_{i}}, i=1, \ldots, r+1$, with each $\varphi_{i}$ having period $2 \pi$. Moreover, we take $C\left(X_{2 r+1}\right)$ to be Gorenstein, meaning that it admits a global holomorphic $(r+1,0)$-form $\Psi_{(r+1,0)}$. For convenience we choose a basis so that this holomorphic volume form has unit charge under $\partial_{\varphi_{1}}$ and is uncharged under $\partial_{\varphi_{i}}, i=2,3, \ldots, r+1$.

The manifold $X_{2 r+1}$ is embedded at $\varrho=1$. The complex structure of the cone pairs the radial vector $\varrho \partial_{\varrho}$ with the Killing vector field $\xi$ tangent to $X_{2 r+1}$, which we may write as

$$
\xi=\sum_{i=1}^{r+1} b_{i} \partial_{\varphi_{i}} .
$$

The vector $\vec{b}=\left(b_{1}, \ldots, b_{r+1}\right)$ then parametrizes the choice of $R$-symmetry vector $\xi$. Notice that we then have

$$
\mathcal{L}_{\xi} \Psi_{(r+1,0)}=\mathrm{i} b_{1} \Psi_{(r+1,0)} \cdot
$$

The complex structure likewise pairs the one-form $\eta$ dual to $\xi$ with $\mathrm{d} \varrho / \varrho$. In particular for Kähler cones

$$
\mathrm{d} \eta=2 \omega_{\text {Sasakian }},
$$

where $\omega_{\text {Sasakian }}$ is the transverse Kähler form. Because $\mathrm{d} \eta$ is also a transverse symplectic form in this case, by definition $\eta$ is a contact one-form on $X_{2 r+1}$. The unique vector field $\xi$ satisfying $\xi\lrcorner \eta=1, \xi\lrcorner \mathrm{d} \eta=0$ is then also called the Reeb vector field. We may write the (Sasakian) metric on $X_{2 r+1}$ as

$$
\mathrm{d} s_{2 r+1}^{2}=\eta^{2}+\mathrm{d} s_{2 r}^{2}(\omega)
$$

where $\mathrm{d} s_{2 r}^{2}(\omega)$ is the transverse Kähler metric with Kähler form $\omega=\omega_{\text {Sasakian. We note }}$ that (3.3) implies that

$$
[\mathrm{d} \eta]=\frac{1}{b_{1}}[\rho] \in H_{B}^{2}\left(\mathcal{F}_{\xi}\right),
$$

where $\mathcal{F}_{\xi}$ is the foliation of $X_{2 r+1}$ induced by the choice of Reeb vector $\xi$, and $\rho$ denotes the Ricci two-form of the transverse Kähler metric $\mathrm{d} s_{2 r}^{2}(\omega)$.

We may next define the moment map coordinates

$$
\left.y_{i} \equiv \frac{1}{2} \varrho^{2} \partial_{\varphi_{i}}\right\lrcorner \eta, \quad i=1, \ldots, r+1 .
$$


These span the so-called moment map polyhedral cone $\mathcal{C} \subset \mathbb{R}^{r+1}$, where the $\vec{y}=\left(y_{1}, \ldots\right.$, $\left.y_{r+1}\right)$ are standard coordinates on $\mathbb{R}^{r+1}$. The polyhedral cone $\mathcal{C}$, which is convex, may be written as

$$
\mathcal{C}=\left\{\vec{y} \in \mathbb{R}^{r+1} \mid\left(\vec{y}, \vec{v}_{a}\right) \geq 0, \quad a=1, \ldots, d\right\},
$$

where $\vec{v}_{a} \in \mathbb{Z}^{r+1}$ are the inward pointing primitive normals to the facets of the polyhedral cone, and the index $a=1, \ldots, d \geq r+1$ labels the facets. Furthermore, $v_{a}=\left(1, w_{a}\right)$, where $w_{a} \in \mathbb{Z}^{r}$, follows from the Gorenstein condition in the basis for $\mathrm{U}(1)^{r+1}$ described above.

As shown in [5], for a Kähler cone metric on $C\left(X_{2 r+1}\right)$ the $R$-symmetry vector $\vec{b}=$ $\left(b_{1}, \ldots, b_{r+1}\right)$ lies in the interior of the Reeb cone, $\vec{b} \in \mathcal{C}_{\text {int }}^{*}$. Here the Reeb cone $\mathcal{C}^{*}$ is defined to be the dual cone to $\mathcal{C}$, with $\mathcal{C}_{\text {int }}^{*}$ being its open interior. Using $\left.\xi\right\lrcorner \eta=1$, together with (3.2) and (3.7), the image of $X_{2 r+1}=\{\varrho=1\}$ under the moment map is then the compact, convex $r$-dimensional polytope

$$
P=P(\vec{b})=\mathcal{C} \cap H(\vec{b}),
$$

where the Reeb hyperplane is by definition

$$
H=H(\vec{b}) \equiv\left\{\vec{y} \in \mathbb{R}^{r+1} \mid(\vec{y}, \vec{b})=\frac{1}{2}\right\}
$$

\subsection{The master volume}

Following [16], we first fix a choice of toric Kähler cone metric on the complex cone $C\left(X_{2 r+1}\right)$. As described in the previous subsection, this allows us to introduce the moment map coordinates $\vec{y}$ in (3.7), together with the angular coordinates $\varphi_{i}, i=1, \ldots, r+1$, as coordinates on $C\left(X_{2 r+1}\right)$. Geometrically, $C\left(X_{2 r+1}\right)$ then fibres over the polyhedral cone $\mathcal{C}$ : over the interior $\mathcal{C}_{\text {int }}$ of $\mathcal{C}$ this is a trivial $\mathrm{U}(1)^{r+1}$ fibration, with the normal vectors $\vec{v}_{a} \in \mathbb{Z}^{r+1}$ to each bounding facet $\left\{\left(\vec{y}, \vec{v}_{a}\right)=0\right\} \subset \partial \mathcal{C}$ specifying which $\mathrm{U}(1) \subset \mathrm{U}(1)^{r+1}$ collapses along that facet. Each such facet is also the image under the moment map of a toric divisor in $C\left(X_{2 r+1}\right)$ - that is, a complex codimension one submanifold that is invariant under the torus $\mathrm{U}(1)^{r+1}$. The index $a=1, \ldots, d$ thus also labels the toric divisors.

For a fixed choice of such complex cone, with Reeb vector $\xi$ given by (3.2), we would then like to study a more general class of transversely Kähler metrics of the form (3.5). In particular, we are interested in the "master volume" defined by

$$
\mathcal{V} \equiv \int_{X_{2 r+1}} \eta \wedge \frac{1}{r !} \omega^{r}
$$

as a function both of the vector $\xi$, and transverse Kähler class $[\omega] \in H_{B}^{2}\left(\mathcal{F}_{\xi}\right)$. Following [16], if we take $c_{a}$ to be basic representatives in $H_{B}^{2}\left(\mathcal{F}_{\xi}\right)$ that lift to integral classes in $H^{2}\left(X_{2 r+1}, \mathbb{Z}\right)$, which are Poincaré dual to the restriction of the toric divisors on $C\left(X_{2 r+1}\right)$, then we can write

$$
[\omega]=-2 \pi \sum_{a=1}^{d} \lambda_{a} c_{a} \in H_{B}^{2}\left(\mathcal{F}_{\xi}\right) .
$$


The $c_{a}$ are not all independent and $[\omega]$ in fact only depends on $d-r$ of the $d$ parameters $\left\{\lambda_{a}\right\}$, as we shall see shortly. It will also be useful to note that the first Chern class of the foliation can be written in terms of the $c_{a}$ as

$$
[\rho]=2 \pi \sum_{a=1}^{d} c_{a} \in H_{B}^{2}\left(\mathcal{F}_{\xi}\right) .
$$

In the special case in which

$$
\lambda_{a}=-\frac{1}{2 b_{1}}, \quad a=1, \ldots d,
$$

we recover the Sasakian Kähler class $[\rho]=2 b_{1}\left[\omega_{\text {Sasakian }}\right]$ and the master volume (3.11) reduces to the Sasakian volume.

Again following [16], the master volume (3.11) may be written as

$$
\mathcal{V}=\frac{(2 \pi)^{r+1}}{|\vec{b}|} \operatorname{vol}(\mathcal{P})
$$

Here the factor of $(2 \pi)^{r+1}$ arises by integrating over the torus $\mathrm{U}(1)^{r+1}$, while $\operatorname{vol}(\mathcal{P})$ is the Euclidean volume of the compact, convex $r$-dimensional polytope

$$
\mathcal{P}=\mathcal{P}\left(\vec{b} ;\left\{\lambda_{a}\right\}\right) \equiv\left\{\vec{y} \in H(\vec{b}) \mid\left(\vec{y}-\vec{y}^{(0)}, \vec{v}_{a}\right) \geq \lambda_{a}, \quad a=1, \ldots, d\right\} .
$$

Here

$$
\vec{y}^{(0)} \equiv\left(\frac{1}{2 b_{1}}, 0, \ldots, 0\right) \in H
$$

which lies in the interior of $\mathcal{P}$, while the $\left\{\lambda_{a}\right\}$ parameters determine the transverse Kähler class. We next introduce the new coordinates

$$
x_{i} \equiv y_{i}-y_{i}^{(0)} .
$$

Notice that the inequalities defining the polytope $\mathcal{P}$ then become simply $\left(\vec{x}, \vec{v}_{a}\right) \geq \lambda_{a}$, $a=1, \ldots, d$, which is the usual way the moment polytope is presented in toric Kähler geometry. In this case $x_{i}$ is the Hamiltonian function for the $i$ th U(1) Killing vector $\partial_{\varphi_{i}}$ with respect to the (transverse) Kähler form $\omega$, i.e. $\left.\mathrm{d} x_{i}=-\partial_{\varphi_{i}}\right\lrcorner \omega$. Using (3.18) we may then also write the master volume (3.15) as an integral

$$
\mathcal{V}=\mathcal{V}\left(\vec{b} ;\left\{\lambda_{a}\right\} ;\left\{\vec{v}_{a}\right\}\right)=(2 \pi)^{r+1} \int_{\mathbb{R}^{r+1}} \prod_{a=1}^{d} \theta\left(\left(\vec{x}, \vec{v}_{a}\right)-\lambda_{a}\right) \delta((\vec{x}, \vec{b})),
$$

where the integration over $\mathbb{R}^{r+1}$ uses the standard Euclidean measure $\mathrm{d} x_{1} \wedge \cdots \wedge \mathrm{d} x_{r+1}$. Here we have emphasized in the notation that the master volume also depends on the choice of polyhedral cone $\mathcal{C}$, via its primitive normal vectors $\vec{v}_{a} \in \mathbb{Z}^{r+1}$, as well as the choice of $R$-symmetry vector $\vec{b}$ and Kähler class parameters $\left\{\lambda_{a}\right\}$. Using (3.19) it is shown in appendix A that $\mathcal{V}$ satisfies the identity

$$
\sum_{a=1}^{d}\left(\vec{v}_{a}-\frac{\vec{b}}{b_{1}}\right) \frac{\partial \mathcal{V}}{\partial \lambda_{a}}=0
$$


meaning that this equation holds for all $\vec{b}$ and $\left\{\lambda_{a}\right\}$ (for fixed polyhedral cone and hence fixed $\left\{\vec{v}_{a}\right\}$ ). It follows that the master volume is invariant under the "gauge" transformations

$$
\lambda_{a} \rightarrow \lambda_{a}+\sum_{i=1}^{r+1} \gamma_{i}\left(v_{a}^{i} b_{1}-b_{i}\right)
$$

for arbitrary constants $\gamma_{i}$, generalizing a result of [19]. For $X_{2 r+1}$, noting that the transformation parametrized by $\gamma_{1}$ is trivial, this explicitly shows that the master volume only depends on $d-r$ of the $d$ parameters $\left\{\lambda_{a}\right\}$.

The master volume $\mathcal{V}$ is homogeneous of degree $r$ in the $\lambda_{a}$, and we have

$$
\mathcal{V} \equiv \int_{X_{2 r+1}} \eta \wedge \frac{1}{r !} \omega^{r}=(-2 \pi)^{r} \sum_{a_{1}, \ldots, a_{r}=1}^{d} \frac{1}{r !} I_{a_{1} \ldots a_{r}} \lambda_{a_{1}} \ldots \lambda_{a_{r}},
$$

where the "intersection numbers" $I_{a_{1} \ldots a_{r}}$ are defined as

$$
I_{a_{1} \ldots a_{r}} \equiv \int_{Y_{2 r+1}} \eta \wedge c_{a_{1}} \wedge \cdots \wedge c_{a_{r}}=\frac{1}{(-2 \pi)^{r}} \frac{\partial^{r} \mathcal{V}}{\partial \lambda_{a_{1}} \ldots \partial \lambda_{a_{r}}}
$$

We may then calculate

$$
\int_{X_{2 r+1}} \eta \wedge \rho^{s} \wedge \frac{1}{(r-s) !} \omega^{r-s}=(-1)^{s} \sum_{a_{1}, \ldots, a_{s}=1}^{d} \frac{\partial^{s} \mathcal{V}}{\partial \lambda_{a_{1}} \ldots \partial \lambda_{a_{s}}}
$$

We also are interested in integrating over $S_{a}$, the $(2 r-1)$-cycle in $X_{2 r+1}$ associated with a toric divisor on the cone and Poincaré dual to $c_{a}$. We have

$$
\begin{aligned}
\int_{S_{a}} \eta \wedge \rho^{s} \wedge \frac{1}{(r-s-1) !} \omega^{r-s-1} & =\int_{Y_{2 r+1}} \eta \wedge \rho^{s} \wedge \frac{1}{(r-s-1) !} \omega^{r-s-1} \wedge c_{a} \\
& =\frac{(-1)^{s+1}}{2 \pi} \sum_{b_{1}, \ldots, b_{s}=1}^{d} \frac{\partial^{s+1} \mathcal{V}}{\partial \lambda_{a} \partial \lambda_{b_{1}} \ldots \partial \lambda_{b_{s}}} .
\end{aligned}
$$

In appendix A we also show that the master volume $\mathcal{V}$ is homogeneous of degree -1 in the $b_{i}$.

It is possible to obtain very explicit formulas for the master volume in low dimensions. In dimensions $r=2$ and $r=3$ the relevant formulae for $X_{5}$ and $X_{7}$ were derived in [16] and [9], respectively. In the present paper we shall also be interested in the case $r=1$, with a three-dimensional toric fibre $X_{3}$. In this case the toric data of a Gorenstein Kähler cone of complex dimension $r+1=2$ is given by the two inward pointing normal vectors $v_{1}=(1,0), v_{2}=(1, p)$, where $p \in \mathbb{N}$. This describes an $A_{p-1}$ singularity, $C\left(X_{3}\right)=\mathbb{C}^{2} / \mathbb{Z}_{p}$, with the $\mathbb{Z}_{p}$ action on $\mathbb{C}^{2}$ given by $\left(z_{1}, z_{2}\right) \mapsto\left(\omega_{p} z_{1}, \omega_{p}^{-1} z_{2}\right)$, where $\omega_{p}$ is a primitive $p$ th root of unity. As shown in appendix $\mathrm{B}$, the master volume of $X_{3}$ in this case is simply

$$
\mathcal{V}\left(\vec{b} ; \lambda_{1}, \lambda_{2} ; \vec{v}_{1}, \vec{v}_{2}\right)=(2 \pi)^{2} \sum_{a=1}^{2}(-1)^{a} \frac{\lambda_{a}}{\left[\vec{v}_{a}, \vec{b}\right]},
$$


where here $\left[\vec{v}_{a}, \vec{b}\right]$ denotes the determinant of the $2 \times 2$ matrix, i.e. $\left[\vec{v}_{a}, \vec{b}\right] \equiv \varepsilon_{i j} v_{a}^{i} b^{j}$. Later in the paper we will also need the master volume in dimension $r=2$. In this case the master volume of $X_{5}$ is [16]

$$
\mathcal{V}\left(\vec{b} ;\left\{\lambda_{a}\right\} ;\left\{\vec{v}_{a}\right\}\right)=\frac{(2 \pi)^{3}}{2} \sum_{a=1}^{d} \lambda_{a} \frac{\lambda_{a-1}\left[\vec{v}_{a}, \vec{v}_{a+1}, \vec{b}\right]-\lambda_{a}\left[\vec{v}_{a-1}, \vec{v}_{a+1}, \vec{b}\right]+\lambda_{a+1}\left[\vec{v}_{a-1}, \vec{v}_{a}, \vec{b}\right]}{\left[\vec{v}_{a-1}, \vec{v}_{a}, \vec{b}\right]\left[\vec{v}_{a}, \vec{v}_{a+1}, \vec{b}\right]},
$$

where $[\cdot, \cdot, \cdot]$ denotes a $3 \times 3$ determinant. Here the facets are ordered anti-clockwise around the polyhedral cone, and we cyclically identify $\vec{v}_{d+1} \equiv \vec{v}_{1}, \vec{v}_{0} \equiv \vec{v}_{d}$, and similarly $\lambda_{d+1} \equiv \lambda_{1}$, $\lambda_{0} \equiv \lambda_{d}$.

Finally, we note that the formulae in this section assume that the polyhedral cone $\mathcal{C}$ is convex, since we started the section with a cone that admits a toric Kähler cone metric. However, as discussed in $[4,16]$, this convexity condition is, in general, too restrictive for applications to the classes of $A d S_{2}$ and $A d S_{3}$ solutions of interest. Indeed, many such explicit supergravity solutions are associated with "non-convex toric cones", as defined in [4], which in particular have toric data which do not define a convex polyhedral cone. As in the above papers and [9], we conjecture that the key formulae in this section are also applicable to non-convex toric cones, and we will assume that this is the case in the sequel. The consistent picture that emerges, combined with similar results in $[4,9,16]$, strongly supports the validity of this conjecture.

\section{Fibred GK geometry}

We would like to study GK geometries of the fibred form (2.18), where the fibres $X_{2 r+1}$ take the toric form described in section 3. In particular, we would like to evaluate the constraint, flux quantization condition and supersymmetric action (2.8), (2.9), (2.10) for these fibred geometries, respectively. In this section we follow a similar analysis to that in section 4 of [16], which studied the case of $X_{5}$ fibred over a Riemann surface $\Sigma_{g}$ of genus $g$. Extending this to $X_{2 r+1}$ fibred over a Kähler base $B_{2 k}$ is relatively straightforward, although for $k>1$ various new features arise compared to the Riemann surface $k=1$ case.

\subsection{General set-up}

The manifolds $X_{2 r+1}$ by definition admit an isometric $\mathrm{U}(1)^{r+1}$ action. We may use this symmetry to fibre $X_{2 r+1}$ over the Kähler base $B_{2 k}$ by picking $r+1 \mathrm{U}(1)$ gauge fields $A_{i}$ on $B_{2 k}, i=1, \ldots, r+1$, with curvatures $F_{i}=\mathrm{d} A_{i}$ given by

$$
\frac{F_{i}}{2 \pi}=\sum_{\alpha} n_{i}^{\alpha} c_{\alpha}^{(2)}
$$

Here $c_{\alpha}^{(2)} \in H^{2}\left(B_{2 k}, \mathbb{R}\right)$ are closed two-forms that generate the free part of $H^{2}\left(B_{2 k}, \mathbb{Z}\right)$, and $n_{i}^{\alpha} \in \mathbb{Z}$. It will be convenient later to take the $c_{\alpha}^{(2)} \in H^{2}\left(B_{2 k}, \mathbb{R}\right)$ to be Poincaré duals to a corresponding basis of $(2 p-2)$-cycles $C_{\alpha}^{(2 p-2)} \in H_{2 p-2}\left(B_{2 k}, \mathbb{Z}\right)$, which by definition means that

$$
\int_{C_{\alpha}^{(2 p-2)}} \Phi=\int_{B_{2 k}} \Phi \wedge c_{\alpha}^{(2)}
$$


holds for all closed ( $2 p-2)$-forms $\Phi$ on $B_{2 k}$. Having chosen the curvatures in (4.1), which amounts to a choice of the integers $n_{i}^{\alpha}$, one then uses the corresponding $\mathrm{U}(1)^{r+1}$ transition functions to fibre $X_{2 r+1}$ over $B_{2 k}$, using the toric action of $\mathrm{U}(1)^{r+1}$ on $X_{2 r+1} \cdot{ }^{2}$

More concretely, the above fibration amounts to a replacement

$$
\mathrm{d} \varphi_{i} \rightarrow \mathrm{d} \varphi_{i}+A_{i} \quad i=1, \ldots, r+1
$$

where recall that $\varphi_{i}$ are the $(2 \pi)$-periodic coordinates on the torus $\mathrm{U}(1)^{r+1}$. As in [16], it is important here to emphasize that the quantities (2.8), (2.9), (2.10) of interest on the total space of the fibration depend only on basic cohomology classes in $H_{B}^{2}\left(\mathcal{F}_{\xi}\right)$. This means that we may use any convenient representative of the various differential forms that enter these quantities - we must only ensure that the representative we use has the correct basic cohomology class.

With these comments in mind, after the fibration the contact one-form $\eta$ on the fibres $X_{2 r+1}$ is effectively replaced by

$$
\eta \rightarrow \eta_{\text {twisted }} \equiv 2 \sum_{i=1}^{r+1} w_{i}\left(\mathrm{~d} \varphi_{i}+A_{i}\right),
$$

where we have defined

$$
\left.\left.w_{i} \equiv y_{i}\right|_{\varrho=1}=\frac{1}{2} \partial_{\varphi_{i}}\right\lrcorner \eta
$$

Recall here that the $y_{i}$ are the moment map coordinates (3.7) on $C\left(X_{2 r+1}\right)$, and $X_{2 r+1}=$ $\{\varrho=1\} \subset C\left(X_{2 r+1}\right)$. We then have

$$
\mathrm{d} \eta_{\text {twisted }}=2 \sum_{i=1}^{r+1} \mathrm{~d} w_{i} \wedge\left(\mathrm{d} \varphi_{i}+A_{i}\right)+2 \sum_{i=1}^{r+1} w_{i} F_{i} .
$$

For the transverse Kähler form $J$ we may write

$$
J=\omega_{\text {twisted }}+J_{B_{2 k}}+\text { basic exact }
$$

up to an irrelevant basic exact form, where $J_{B_{2 k}}$ is a Kähler form on the base $B_{2 k}$ and

$$
\omega_{\text {twisted }} \equiv \sum_{i=1}^{r+1} \mathrm{~d} x_{i} \wedge\left(\mathrm{d} \varphi_{i}+A_{i}\right)+\sum_{i=1}^{r+1} x_{i} F_{i} .
$$

Here we have identified

$$
\left.\mathrm{d} x_{i}=-\partial_{\varphi_{i}}\right\lrcorner \omega
$$

so that the $x_{i}$ are global Hamiltonian functions on the fibre $X_{2 r+1}$, invariant under the torus action, $c f$. the discussion after equation (3.18), where the same functions $x_{i}$ appear. Notice that these are a priori defined only up to an additive constant, but that via equation (4.7)

\footnotetext{
${ }^{2}$ Notice that a choice of $n_{i}^{\alpha} \in \mathbb{Z}$ only determines the principal $\mathrm{U}(1)^{r+1}$ bundle up to a torsion class in $H^{2}\left(B_{2 k}, \mathbb{Z}\right)$, although this torsion data will not enter the formulae that follow.
} 
such a constant shift may be absorbed into a redefinition of the Kähler form $J_{B_{2 k}}$. As in (4.1) we may then similarly decompose this Kähler form on the base as

$$
J_{B_{2 k}}=\sum_{\alpha} a^{\alpha} c_{\alpha}^{(2)}
$$

where $a_{\alpha} \in \mathbb{R}$.

At the level of the formulae (2.8), (2.9), (2.10), which are expressed as integrals on the total space of the fibration, we may then simply substitute

$$
\begin{aligned}
J & \rightarrow \omega+x_{i} F_{i}+J_{B_{2 k}}, \\
\eta & \rightarrow \eta \\
\mathrm{d} \eta & \rightarrow \mathrm{d} \eta+2 w_{i} F_{i}
\end{aligned}
$$

where on the right hand side, in a slight abuse of notation, $\omega, \eta$ and $\mathrm{d} \eta$ are quantities on the fibre $X_{2 r+1}$, while the remaining terms are quantities on the base $B_{2 k}$.

The holomorphic $(r+1,0)$-form $\Psi_{(r+1,0)}$ on the cone $C\left(X_{2 r+1}\right)$ over the fibre has unit charge under $\partial_{\varphi_{1}}$, meaning there is an explicit $\mathrm{e}^{\mathrm{i} \varphi_{1}}$ dependence, where recall that we have chosen the basis for the torus action so that this is the case. On the other hand, the holomorphic $(n+1,0)$-form $\Psi$ on $C\left(Y_{2 n+1}\right)$ is constructed by taking the wedge product of the canonical holomorphic $(k, 0)$-form on the Kähler base $B_{2 k}$ with the $(r+1,0)$-form $\Psi_{(r+1,0)}$ on the fibre, twisting the latter using the $r+1$ line bundles over $k$ with curvatures $F_{i}, i=1, \ldots, r+1$. The canonical $(k, 0)$-form on the Kähler base $B_{2 k}$ is not globally defined in general (unless the base is Calabi-Yau), being a section of the canonical line bundle $K_{B_{2 k}}$. However, due to the twisting, $\mathrm{e}^{\mathrm{i} \varphi_{1}}$ is precisely a section of the line bundle over $B_{2 k}$ with first Chern class $\left[F_{1} / 2 \pi\right] \in H^{2}\left(B_{2 k}, \mathbb{Z}\right)$. Neither section exists globally in general, but the wedge product does have a global nowhere zero section, and hence gives rise to a global $(n+1,0)$-form $\Psi$ on $C\left(Y_{2 n+1}\right)$, precisely if

$$
\left[\frac{F_{1}}{2 \pi}\right]=-c_{1}\left(K_{B_{2 k}}\right)=c_{1}\left(B_{2 k}\right) \text {. }
$$

When this condition holds, the cone $C\left(Y_{2 n+1}\right)$ has a global $(n+1,0)$-form. The condition (4.12) generalizes the twist condition over a Riemann surface (where $k=1$ ) presented in $[9,16]$.

Finally, recalling (3.3) and (2.7), for $Y_{2 r+2 k+1}$ we need to take

$$
b_{1}=\frac{2}{r+k-2} .
$$

In the expressions given in the next subsections, it is important that this condition is only imposed after taking any derivatives with respect to the $b_{i}$.

In the remainder of this section we simply present the final formulae for various lowdimensional cases of interest, referring to the appendices for further details of the calculations involved. 


\section{$4.2 \quad X_{2 r+1} \hookrightarrow Y_{2 r+3} \rightarrow B_{2}$}

Here $r=2$ and $r=3$ are relevant for the type IIB case and the $D=11$ case, respectively. In fact these two cases were already treated in [16] and [9], respectively. Generalizing the calculations to general $r \geq 2$ is straightforward.

We begin by noting that the topological constraint (2.8) can be written in the form

$$
\sum_{a, b=1}^{d} \frac{\partial^{2} \mathcal{V}}{\partial \lambda_{a} \partial \lambda_{b}} \operatorname{vol}\left(B_{2}\right)+b_{1} \sum_{a=1}^{d} \sum_{i=1}^{r+1} \frac{\partial^{2} \mathcal{V}}{\partial \lambda_{a} \partial b_{i}} \int_{B_{2}} F_{i}-\sum_{a=1}^{d} \frac{\partial \mathcal{V}}{\partial \lambda_{a}} \int_{B_{2}} F_{1}=0
$$

Next we consider the flux quantization conditions given in (2.9). There are two classes of $(2 r+1)$-cycles to consider. First, there is the distinguished $(2 r+1)$-cycle, $\Sigma$, obtained by picking a point on the Kähler base $B_{2}$. We find

$$
-\sum_{a=1}^{d} \frac{\partial \mathcal{V}}{\partial \lambda_{a}}=\nu_{r+1} N
$$

where we recall that $\nu_{r+1}$ is a non-zero, real constant, fixed for the case of $r=2,3$ as in (2.12), (2.16), respectively, and $N \in \mathbb{Z}$. The second class of $(2 r+1)$-cycles are given by the total spaces $\Sigma_{a}$ of the fibrations

$$
S_{a} \hookrightarrow \Sigma_{a} \rightarrow B_{2},
$$

where $S_{a}$ is a $(2 r-1)$-cycle in $X_{2 r+1}$ associated with a toric divisor on the associated cone $C\left(X_{2 r+1}\right)$. For these cycles we have

$$
\frac{1}{2 \pi} \sum_{b=1}^{d} \frac{\partial^{2} \mathcal{V}}{\partial \lambda_{a} \partial \lambda_{b}} \operatorname{vol}\left(B_{2}\right)+\frac{b_{1}}{2 \pi} \sum_{i=1}^{r+1} \frac{\partial^{2} \mathcal{V}}{\partial \lambda_{a} \partial b_{i}} \int_{B_{2}} F_{i}=\nu_{r+1} M_{a},
$$

with $M_{a} \in \mathbb{Z}$. The $S_{a}$ are not linearly independent cycles in the fibre $X_{2 r+1}$, which leads to the corresponding linear relations among the flux numbers $[9,16]$ :

$$
\sum_{a=1}^{d} v_{a}^{i} M_{a}=-N \int_{B_{2}} \frac{F_{i}}{2 \pi}, \quad i=1, \ldots, r+1 .
$$

In the above expressions, from (4.13), we should take

$$
b_{1}=\frac{2}{r-1},
$$

after taking derivatives with respect to $b_{i}$. Finally, the supersymmetric action, given in (2.10), can be cast in the form

$$
S_{\mathrm{SUSY}}=\nu_{r+1} \frac{2 \pi}{r}\left(\frac{N}{2 \pi} \operatorname{vol}\left(B_{2}\right)-\sum_{a=1}^{d} \lambda_{a} M_{a}\right)
$$




\section{$4.3 \quad X_{2 r+1} \hookrightarrow Y_{2 r+5} \rightarrow B_{4}$}

Now $r=1$ is relevant for the type IIB case, while $r=2$ is relevant for the $D=11$ case.

The topological constraint condition (2.8) is given by

$$
\begin{aligned}
& \sum_{a, b=1}^{d} \frac{\partial^{2} \mathcal{V}}{\partial \lambda_{a} \partial \lambda_{b}} \operatorname{vol}\left(B_{4}\right)+b_{1} \sum_{i=1}^{r+1} \sum_{a=1}^{d} \frac{\partial^{2} \mathcal{V}}{\partial \lambda_{a} \partial b_{i}} \int_{B_{4}} F_{i} \wedge J_{B_{4}}-\sum_{a=1}^{d} \frac{\partial \mathcal{V}}{\partial \lambda_{a}} \int_{B_{4}} F_{1} \wedge J_{B_{4}} \\
& \quad+b_{1}^{2} \sum_{i, j=1}^{r+1} \frac{\partial^{2} \mathcal{V}}{\partial b_{i} \partial b_{j}} \int_{B_{4}} \frac{1}{2} F_{i} \wedge F_{j}=0 .
\end{aligned}
$$

There are two types of flux integrals, corresponding to two types of $(2 r+3)$-cycles. The first type of cycles have the fibred form

$$
X_{2 r+1} \hookrightarrow \Sigma_{\alpha} \rightarrow C_{\alpha}^{(2)},
$$

with $C_{\alpha}^{(2)} \subset B_{4}$ a two-cycle. We find

$$
-\sum_{a=1}^{d} \frac{\partial \mathcal{V}}{\partial \lambda_{a}} \int_{C_{\alpha}^{(2)}} J_{B_{4}}-b_{1} \sum_{i=1}^{r+1} \frac{\partial \mathcal{V}}{\partial b_{i}} \int_{C_{\alpha}^{(2)}} F_{i}=\nu_{r+2} N_{\alpha}
$$

where $\nu_{r+2}$ is a non-zero, real constant, fixed for the case of $r=1,2$ as in (2.12), (2.16), respectively, and $N_{\alpha} \in \mathbb{Z}$. The second set of $(2 r+3)$-cycles have the fibred form

$$
S_{a} \hookrightarrow \Sigma_{a} \rightarrow B_{4},
$$

where $S_{a}$ is a $(2 r-1)$-cycle in $X_{2 r+1}$ associated with a toric divisor on the associated cone $C\left(X_{2 r+1}\right)$. We find

$$
\begin{aligned}
\frac{1}{2 \pi} \sum_{b=1}^{d} \frac{\partial^{2} \mathcal{V}}{\partial \lambda_{a} \partial \lambda_{b}} \operatorname{vol}\left(B_{4}\right) & +\frac{b_{1}}{2 \pi} \sum_{i=1}^{r+1} \frac{\partial^{2} \mathcal{V}}{\partial \lambda_{a} \partial b_{i}} \int_{B_{4}} F_{i} \wedge J_{B_{4}} \\
& -\frac{b_{1}}{2 \pi} \sum_{i_{1}, i_{2}=1}^{r+1} \frac{\partial^{2} \mathcal{V}}{\partial b_{i_{2}} \partial v_{a}^{i_{1}}} \int_{B_{4}} \frac{1}{2} F_{i_{1}} \wedge F_{i_{2}}=\nu_{r+2} M_{a}
\end{aligned}
$$

with $M_{a} \in \mathbb{Z}$. Again, the $S_{a}$ are not linearly independent cycles in the fibre: multiplying (4.25) by $v_{a}^{i}$ and summing over $a=1, \ldots, d$, and using (4.21), (4.23) and the identity (3.20), one can show that

$$
\sum_{a=1}^{d} v_{a}^{i} M_{a}=-\sum_{\alpha} N_{\alpha} n_{i}^{\alpha}
$$

where the twisting parameters $n_{i}^{\alpha}$ were introduced in (4.1). In the above expressions, from (4.13), we should take

$$
b_{1}=\frac{2}{r},
$$


after taking derivatives with respect to $b_{i}$. Finally, the supersymmetric action (2.10) can be written as

$$
S_{\mathrm{SUSY}}=\nu_{r+2} \frac{2 \pi}{r+1}\left(\frac{1}{2 \pi} a^{\alpha} N_{\alpha}-\sum_{a=1}^{d} \lambda_{a} M_{a}\right),
$$

where recall that the $a^{\alpha}$ were introduced in (4.10) and parametrize the Kähler form, $J_{B_{2 k}}$, on the base $B_{4}$.

\section{$4.4 \quad X_{2 r+1} \hookrightarrow Y_{2 r+7} \rightarrow B_{6}$}

Now $r=0$ is relevant ${ }^{3}$ for the type IIB case while $r=1$ is relevant for the $D=11$ case.

The topological constraint condition (2.8) is given by

$$
\begin{aligned}
\sum_{a, b=1}^{d} & \frac{\partial^{2} \mathcal{V}}{\partial \lambda_{a} \partial \lambda_{b}} \operatorname{vol}\left(B_{6}\right)+b_{1} \sum_{i=1}^{r+1} \sum_{a=1}^{d} \frac{\partial^{2} \mathcal{V}}{\partial \lambda_{a} \partial b_{i}} \int_{B_{6}} F_{i} \wedge \frac{1}{2} J_{B_{6}}^{2}-\sum_{a=1}^{d} \frac{\partial \mathcal{V}}{\partial \lambda_{a}} \int_{B_{6}} F_{1} \wedge \frac{1}{2} J_{B_{6}}^{2} \\
& +b_{1}^{2} \sum_{i, j=1}^{r+1} \frac{\partial^{2} \mathcal{V}}{\partial b_{i} \partial b_{j}} \int_{B_{6}} \frac{1}{2} F_{i} \wedge F_{j} \wedge J_{B_{6}} \\
& -b_{1}^{2} \sum_{i_{1}, i_{2}, i_{3}=1}^{r+1} \frac{\partial^{2}}{\partial b_{i_{2}} \partial b_{i_{3}}}\left(\frac{1}{r+1} \sum_{a=1}^{d} \lambda^{a} \frac{\partial \mathcal{V}}{\partial v_{a}^{i_{1}}}\right) \int_{B_{6}} \frac{1}{3 !} F_{i_{1}} \wedge F_{i_{2}} \wedge F_{i_{3}}=0
\end{aligned}
$$

There are two types of flux integrals, corresponding to two types of $(2 r+5)$-cycles. The first type of cycles have the fibred form

$$
X_{2 r+1} \hookrightarrow \Sigma_{\alpha} \rightarrow C_{\alpha}^{(4)},
$$

with $C_{\alpha}^{(4)} \subset B_{6}$ a four-cycle. We find

$$
\begin{aligned}
& -\sum_{a=1}^{d} \frac{\partial \mathcal{V}}{\partial \lambda_{a}} \int_{C_{\alpha}^{(4)}} \frac{1}{2} J_{B_{6}}^{2}-b_{1} \sum_{i=1}^{r+1} \frac{\partial \mathcal{V}}{\partial b_{i}} \int_{C_{\alpha}^{(4)}} F_{i} \wedge J_{B_{6}} \\
& +\frac{b_{1}}{r+1} \sum_{a=1}^{d} \sum_{i_{1}, i_{2}=1}^{r+1} \lambda^{a} \frac{\partial^{2} \mathcal{V}}{\partial b_{i_{2}} \partial v_{a}^{i_{1}}} \int_{C_{\alpha}^{(4)}} \frac{1}{2} F_{i_{1}} \wedge F_{i_{2}}=\nu_{r+3} N_{\alpha}
\end{aligned}
$$

where $\nu_{r+3}$ is a non-zero, real constant, fixed for the case of $r=0,1$ as in (2.12), (2.16), respectively, and $N_{\alpha} \in \mathbb{Z}$. The second set of $(2 r+5)$-cycles are given by

$$
S_{a} \hookrightarrow \Sigma_{a} \rightarrow B_{6},
$$

\footnotetext{
${ }^{3}$ When $r=0$ the fibre $X_{1}$ is simply a circle: we have only one toric vector $v_{1}=1$, no Kähler parameters $\lambda_{a}$, and the master volume is simply $\mathcal{V}=2 \pi / b_{1}$. There is only one twisting $\mathrm{U}(1)$ bundle with curvature $F_{1}$, and moreover from (4.12) we have $\left[F_{1}\right]=[\rho]$. In this case, the only terms which contribute are those involving only derivatives of $\mathcal{V}$ with respect to $b_{1}$. The formulae in this subsection then simply give rise to the formulae (2.8)-(2.9) for the case of a regular $\mathrm{U}(1)$ fibration over $B_{6}$ with $\eta=\frac{1}{2}\left(\mathrm{~d} \varphi_{1}+P\right)$.
} 
where $S_{a}$ is a $(2 r-1)$-cycle in $X_{2 r+1}$ associated with a divisor on the cone $C\left(X_{2 r+1}\right)$. We find

$$
\begin{aligned}
\frac{1}{2 \pi} \sum_{b=1}^{d} & \frac{\partial^{2} \mathcal{V}}{\partial \lambda_{a} \partial \lambda_{b}} \operatorname{vol}\left(B_{6}\right)+\frac{b_{1}}{2 \pi} \sum_{i=1}^{r+1} \frac{\partial^{2} \mathcal{V}}{\partial \lambda_{a} \partial b_{i}} \int_{B_{6}} F_{i} \wedge \frac{1}{2} J_{B_{6}}^{2} \\
& -\frac{b_{1}}{2 \pi} \sum_{i_{1}, i_{2}=1}^{r+1} \frac{\partial^{2} \mathcal{V}}{\partial b_{i_{2}} \partial v_{a}^{i_{1}}} \int_{B_{6}} \frac{1}{2} F_{i_{1}} \wedge F_{i_{2}} \wedge J_{B_{6}} \\
& +\frac{b_{1}}{2 \pi} \sum_{i_{1}, i_{2}, i_{3}=1}^{r+1} \frac{\partial}{\partial b_{i_{3}}}\left(\frac{1}{(r+1)} \sum_{b_{1}=1}^{d} \lambda^{b_{1}} \frac{\partial^{2} \mathcal{V}}{\partial v_{b_{1}}^{i_{1}} \partial v_{a}^{i_{2}}}\right) \int_{B_{6}} \frac{1}{3 !} F_{i_{1}} \wedge F_{i_{2}} \wedge F_{i_{3}}=\nu_{r+3} M_{a}
\end{aligned}
$$

with $M_{a} \in \mathbb{Z}$. Again, the $S_{a}$ are not linearly independent cycles in the fibre: multiplying (4.25) by $v_{a}^{i}$ and summing over $a=1, \ldots, d$, and using (4.29), (4.31) one can show that

$$
\sum_{a=1}^{d} v_{a}^{i} M_{a}=-\sum_{\alpha} N_{\alpha} n_{i}^{\alpha},
$$

where the $n_{i}^{\alpha}$ were introduced in (4.1). In proving this, we have also used the identity (A.16) in appendix A. In the above expressions, from (4.13), we should take

$$
b_{1}=\frac{2}{r+1},
$$

after taking derivatives with respect to $b_{i}$. Finally, the supersymmetric action (2.10) can be written as

$$
S_{\mathrm{SUSY}}=\nu_{r+3} \frac{2 \pi}{r+2}\left(\frac{1}{2 \pi} a^{\alpha} N_{\alpha}-\sum_{a=1}^{d} \lambda_{a} M_{a}\right),
$$

where the $a^{\alpha}$ were introduced in (4.10) and parametrize the Kähler form, $J_{B_{6}}$, on the base $B_{6}$.

\section{Examples}

In this section we illustrate our general formalism and procedure in a variety of examples, focusing on the cases where the base space $B_{2 k}$ has complex dimension $k=2$ and $k=$ $3{ }^{4}$ In addition to reproducing the results of some known explicit supergravity solutions summarized in appendix D, where the bases $B_{4}$ and $B_{6}$ are Kähler-Einstein manifolds, we also work out examples where the base manifold is Kähler, but not Einstein. In particular, we present the calculations for $B_{4}=\Sigma_{g_{1}} \times \Sigma_{g_{2}}$, namely the product of two Riemann surfaces of genus $g_{1}$ and $g_{2}$, as well as for $B_{4}=\mathbb{F}_{n}$, the $n$th Hirzebruch surface.

\subsection{Type IIB}

In this subsection we consider $A d S_{3} \times Y_{7}$ examples of the form $X_{3} \hookrightarrow Y_{7} \rightarrow B_{4}$, for a variety of Kähler cases $B_{4}$.

\footnotetext{
${ }^{4}$ The Riemann surface case $k=1$ has already been treated extensively in $[9,10,16,19]$.
} 


\subsection{1 $\quad B_{4}=d P_{k}$}

We begin with the case that $B_{4}=d P_{k}$, the $k$ th del Pezzo surface. ${ }^{5}$ By definition this is the complex projective space $\mathbb{C} P^{2}$ blown up at $k=0, \ldots, 8$ generic points. We let

$$
c_{1}=3 H-\sum_{i=1}^{k} E_{i}
$$

denote the anti-canonical class, where $H$ is the hyperplane class, and $E_{i}$ denote the exceptional divisors in the blow-up. We denote $M^{(k)} \equiv \int_{d P_{k}} c_{1} \wedge c_{1}=9-k$.

For simplicity we will here only present the special case where the cohomology classes of the Kähler form $J_{B_{4}}$ and curvatures of the fibration $F_{i}$ in $H^{2}\left(d P_{k}, \mathbb{R}\right)$ are proportional to the class $c_{1}$. We thus write

$$
\left[J_{B_{4}}\right]=A \frac{c_{1}}{m_{k}}, \quad \frac{1}{2 \pi}\left[F_{i}\right]=n_{i} \frac{c_{1}}{m_{k}},
$$

where $i=1,2$ and $A \in \mathbb{R}, n_{i} \in \mathbb{Z}$. Here $m_{k}$ is the Fano index of $d P_{k}$. By definition this is the largest positive integer so that $c_{1} / m_{k} \in H^{2}\left(d P_{k}, \mathbb{Z}\right)$ is an integer class. This is $m_{0}=3$ for $\mathbb{C} P^{2}$, but $m_{k}=1$ for the remaining del Pezzo surfaces $d P_{k}$ with $k=1, \ldots, 8$. Furthermore, we note from (4.12) that we have $n_{1}=m_{k}$. This case then has three flux quantum numbers: $N, M_{i}$, for $i=1,2$. In particular the two-cycle $C^{(2)}$ for flux quantum number $N$ in (4.23) is taken to be the Poincaré dual to $c_{1} / m_{k}$.

We first solve the constraint equation (4.21) for $A$ to obtain

$$
A=\frac{2 \pi\left(n_{2}^{2} \lambda_{1}\left(2 p-b_{2}\right)^{3}+b_{2}^{3} \lambda_{2}\left(m_{k} p-n_{2}\right)^{2}\right)}{b_{2} p\left(2 p-b_{2}\right)\left(2 n_{2}\left(p-b_{2}\right)+m_{k} b_{2} p\right)}
$$

where here, as below, we have set $b_{1}=2$ as required for an $A d S_{3}$ solution after taking derivatives with respect to the $b_{i}$. We then solve the expression for the preferred flux, $N$, in (4.23) for one of the transverse Kähler class parameters $\lambda_{a}$, specifically $\lambda_{1}$, to obtain

$$
\lambda_{1}=\frac{b_{2} \lambda_{2}}{b_{2}-2 p}-\frac{m_{k}^{2} b_{2}\left[2 n_{2}\left(p-b_{2}\right)+m_{k} b_{2} p\right]}{16 \pi^{3} M^{(k)} n_{2}\left(m_{k} p-n_{2}\right)} \nu_{3} N
$$

We then find that the two remaining fluxes can be expressed as

$$
M_{1}=\frac{\left(n_{2}-m_{k} p\right)}{p} N, \quad M_{2}=-\frac{n_{2}}{p} N
$$

while the off-shell trial central charge function, given by (2.13) and (4.28), takes the form

$$
\mathscr{Z}=-\frac{3 m_{k}^{2}\left[n_{2}^{2}\left(4 p^{2}-6 b_{2} p+3 b_{2}^{2}\right)+m_{k} b_{2} n_{2} p\left(2 p-3 b_{2}\right)+m_{k}^{2} b_{2}^{2} p^{2}\right]}{M^{(k)} n_{2} p\left(m_{k} p-n_{2}\right)} N^{2} .
$$

\footnotetext{
${ }^{5}$ In the rest of the paper the base space has been denoted $B_{2 k}$, of complex dimension $k$. In this section this $k=2$, and in an abuse of notation instead in this subsection the integer $k=0, \ldots, 8$ will label the del Pezzo surface $d P_{k}$.
} 
Extremizing $\mathscr{Z}$ over $b_{2}$ we find

$$
b_{2}=\frac{n_{2} p\left(3 n_{2}-m_{k} p\right)}{m_{k}^{2} p^{2}-3 m_{k} n_{2} p+3 n_{2}^{2}},
$$

and hence $\left.c_{\text {sugra }} \equiv \mathscr{Z}\right|_{\text {on-shell }}$ is given by

$$
c_{\text {sugra }}=\frac{9 m_{k}^{2} n_{2} p\left(n_{2}-m_{k} p\right)}{M^{(k)}\left(m_{k}^{2} p^{2}-3 m_{k} n_{2} p+3 n_{2}^{2}\right)} N^{2} .
$$

We can now compare with the explicit $A d S_{3} \times \mathscr{Y}^{\mathrm{p}, \mathrm{q}}\left(\mathrm{KE}_{4}^{+}\right)$supergravity solutions of [17], which are briefly summarized in appendix D.1. For each choice of $\mathrm{KE}_{4}^{+}$these solutions are specified by two positive, relatively prime integers $p>0, q>0$, as well as an overall flux number $n$. We make the obvious identifications $M=M^{(k)}$ and $m=m_{k}$, together with $\mathrm{p}=-n_{2}, \mathrm{q}=p$ and $n=\left(m_{k}^{2} h / p M^{(k)}\right) N$, where $h \equiv \operatorname{hcf}\left(M^{(k)} / m_{k}^{2}, p\right)$. We then notice that the flux quantum numbers can be written as

$$
M_{1}=\left(n_{2}-m_{k} p\right) \frac{M^{(k)}}{h m_{k}^{2}} n, \quad M_{2}=-n_{2} \frac{M^{(k)}}{h m_{k}^{2}} n, \quad N=\frac{M^{(k)}}{m_{k}^{2}} \frac{p}{h} n .
$$

Each term in the products is manifestly an integer, provided that $n$ is an integer, ensuring that $M_{1}, M_{2}, N \in \mathbb{Z}$. Moreover, this ensures that all flux quantum numbers are integer. To see this, recall from (5.2) that $\left[J_{B_{4}}\right]$ and $\left[F_{i}\right]$ are both proportional to the class $c_{1}$. From the general flux quantization condition (4.23), recalling that $N$ is the flux through the two-cycle Poincaré dual to $c_{1} / m_{k}$ as well as the expression for $N$ in (5.9), we may then deduce that the flux associated to an arbitrary two-cycle $C_{\alpha}^{(2)} \subset d P_{k}$ is

$$
N_{\alpha}=\frac{p}{h} n \int_{C_{\alpha}^{(2)}} \frac{c_{1}}{m_{k}} \in \mathbb{Z}
$$

All flux quantum numbers are hence integer, provided that $n$ is an (arbitrary) integer. We find that the fluxes of the explicit supergravity solutions, summarized in (D.1), are related to the $M_{i}$ via $N\left(D_{0}\right)=M_{1}$ and $N\left(\tilde{D}_{0}\right)=-M_{2}$. Finally, the expression for the central charge, given in (5.8) precisely agrees with the expression obtained from the explicit supergravity solution (D.2). We shall return to comment on the formula (5.8) for $k=1,2$, where no Kähler-Einstein metric exists, in subsection 5.1.3.

\subsection{2 $\quad B_{4}=\Sigma_{g_{1}} \times \Sigma_{g_{2}}$}

We next examine the case when $B_{4}=\Sigma_{g_{1}} \times \Sigma_{g_{2}}$ is a product of two Riemann surfaces of genus $g_{1}$ and $g_{2}$. We introduce the normalized volume form classes $\mathrm{vol}_{1}$, $\mathrm{vol}_{2}$ for each Riemann surface, respectively, where $\int_{\Sigma_{g_{1}}} \operatorname{vol}_{1}=1=\int_{\Sigma_{g_{2}}}$ vol $_{2}$. We may then write

$$
\left[J_{B_{4}}\right]=A_{1} \mathrm{vol}_{1}+A_{2} \mathrm{vol}_{2}, \quad \frac{1}{2 \pi}\left[F_{i}\right]=n_{i} \mathrm{vol}_{1}+k_{i} \mathrm{vol}_{2},
$$

where $A_{1}, A_{2} \in \mathbb{R}$ and $n_{i}, k_{i} \in \mathbb{Z}, i=1,2$. We note from (4.12) that we have $n_{1}=2-2 g_{1}$, $k_{1}=2-2 g_{2}$. This case then has four flux quantum numbers: $N_{i}, M_{i}$, for $i=1,2$. 
As in the previous subsection we first solve the constraint equation (4.21), where we choose to eliminate the Kähler class parameter $A_{1}$. We then solve the expression for the fluxes $N_{i}$ given by (4.23), where $i=1,2$ labels the Riemann surfaces, and eliminate $A_{2}$ and $\lambda_{1}$. We then find that two remaining fluxes can be expressed as

$$
M_{1}=\frac{\left[2\left(g_{1}-1\right) p+n_{2}\right] N_{2}+\left[2\left(g_{2}-1\right) p+k_{2}\right] N_{1}}{p}, \quad M_{2}=-\frac{k_{2} N_{1}+n_{2} N_{2}}{p},
$$

while the off-shell trial entral charge function, given by (2.13) and (4.28), may be computed as a function of $b_{2}$, and depends on the parameters $p, g_{1}, g_{2}, n_{2}, k_{2}, N_{1}, N_{2}$. This may then be extremized over $b_{2}$ and evaluated on-shell. Rather than give the general expressions, which are rather unwieldy, we here present the special symmetric case where we choose $k_{2}=n_{2} \equiv k$ and $N_{2}=N_{1} \equiv N$. We then find that the extremal value of $b_{2}$ is given by

$$
b_{2}=\frac{k p\left[p\left(g_{1}+g_{2}-2\right)+3 k\right]}{3 k p\left(g_{1}+g_{2}-2\right)+p^{2}\left(g_{1}+g_{2}-2\right)^{2}+3 k^{2}},
$$

while the central charge is

$$
c_{\text {sugra }}=\frac{18 k p\left[p\left(g_{1}+g_{2}-2\right)+k\right]}{3 k p\left(g_{1}+g_{2}-2\right)+p^{2}\left(g_{1}+g_{2}-2\right)^{2}+3 k^{2}} N^{2} .
$$

Setting $g_{1}=g_{2}=0$, which corresponds to $B_{4}=S^{2} \times S^{2}$, we find that the central charge (5.14) matches the explicit supergravity solution result in (D.2), where $m=2$, $M=8$, and we identify parameters as $\mathrm{p}=-k, \mathrm{q}=p$ and $n=(h / p) N$. In particular we also then find that the fluxes of the explicit supergravity solutions, summarized in (D.1), are related to the $M_{i}$ via $N\left(D_{0}\right)=M_{1}$ and $N\left(\tilde{D}_{0}\right)=-M_{2}$.

\subsection{3 $\quad B_{4}=\mathbb{F}_{n}$}

Finally, we examine the case when $B_{4}=\mathbb{F}_{n}$ is the $n$th Hirzebruch surface. This is the complex surface defined as the total space of a $\mathbb{C} P^{1}$ bundle over $\mathbb{C} P^{1}$. There are various equivalent ways to describe the fibration. For example, one can take the complex line bundle $\mathcal{O}(-n)$ over $\mathbb{C} P^{1}$, and add a point at infinity to each fibre to make the fibres Riemann spheres $\mathbb{C} \cup\{\infty\} \cong \mathbb{C} P^{1}$. Alternatively, one can take the projectivization $\mathbb{P}(\mathcal{O}(0) \oplus \mathcal{O}(-n))$. In the first description, we shall refer to the origin of the complex line fibre as the south pole of the Riemann sphere, and the point at infinity that we add as the north pole. These give rise to sections $S_{1}, S_{2}$ of the $\mathbb{C} P^{1}$ bundle over $\mathbb{C} P^{1}$, respectively, which have intersection numbers $S_{1} \cdot S_{1}=n, S_{2} \cdot S_{2}=-n, S_{1} \cdot S_{2}=0$. Another natural two-cycle is the class $F$ of the fibre, at a fixed point on the $\mathbb{C} P^{1}$ base. This clearly has intersection numbers $F \cdot S_{1}=1=F \cdot S_{2}, F \cdot F=0$. A convenient basis of two-cycles for $H_{2}\left(\mathbb{F}_{n}, \mathbb{Z}\right) \cong \mathbb{Z}^{2}$ is then $\left\{F, S_{1}\right\}$. With respect to this basis, the above formulae imply that the intersection form is

$$
I=\left(\begin{array}{ll}
0 & 1 \\
1 & n
\end{array}\right) .
$$


We denote the Poincaré dual two-form basis for $\left\{F, S_{1}\right\}$ as $\left\{e_{1}=\hat{F}, e_{2}=\hat{S}_{1}\right\}$. These form a dual basis for the cohomology $H^{2}\left(\mathbb{F}_{n}, \mathbb{Z}\right) \cong \mathbb{Z}^{2}$, where

$$
\int_{\mathbb{F}_{n}} e_{\alpha} \wedge e_{\beta}=I_{\alpha \beta}, \quad \alpha, \beta=1,2
$$

With this notation in hand, we may then write the cohomology classes of the Kähler form $J_{B_{4}}$ and curvature two-forms $F_{i}$ in $H^{2}\left(\mathbb{F}_{n}, \mathbb{R}\right)$ as

$$
\left[J_{B_{4}}\right]=\sum_{\alpha=1}^{2} A_{\alpha} e_{\alpha}, \quad \frac{1}{2 \pi}\left[F_{i}\right]=\mathrm{n}_{i} e_{1}+\mathrm{m}_{i} e_{2},
$$

where $A_{\alpha} \in \mathbb{R}, \mathrm{n}_{i}, \mathrm{~m}_{i} \in \mathbb{Z}$ and $i=1,2, \alpha=1,2$. The anti-canonical class of $\mathbb{F}_{n}$ is given by

$$
c_{1}\left(\mathbb{F}_{n}\right)=2 \hat{F}+\hat{S}_{1}+\hat{S}_{2}=(2-n) \hat{F}+2 \hat{S}_{1}=(2-n) e_{1}+2 e_{2},
$$

where in the second step we have used the fact that $n F=S_{1}-S_{2}$, with the same linear relation of course holding for the Poincaré duals. From equation (4.12) we thus deduce that

$$
\mathrm{n}_{1}=2-n, \quad \mathrm{~m}_{1}=2 .
$$

As in the previous subsection we first solve the constraint equation (4.21), where we choose to eliminate the Kähler class parameter $A_{1}$. We then solve the expression for the fluxes $N_{\alpha}$ given by (4.23), where $\alpha=1,2$ labels the basis two-cycles in $\mathbb{F}_{n}$, and eliminate $A_{2}$ and $\lambda_{1}$. We then find that two remaining fluxes can be expressed as

$$
M_{1}=\frac{N_{1}\left[(n-2) p+\mathrm{n}_{2}\right]+N_{2}\left(\mathrm{~m}_{2}-2 p\right)}{p}, \quad M_{2}=-\frac{\mathrm{n}_{2} N_{1}+\mathrm{m}_{2} N_{2}}{p} .
$$

The off-shell trial entral charge function, given by (2.13) and (4.28), may be computed as a function of $b_{2}$, and depends on the parameters $p, n, \mathrm{n}_{2}, \mathrm{~m}_{2}, N_{1}, N_{2}$. This may then be extremized over $b_{2}$ and evaluated on-shell, to obtain the central charge

$$
\begin{aligned}
c_{\text {sugra }}= & 3 N_{1} p\left(n N_{1}-2 N_{2}\right)\left\{\mathrm { m } _ { 2 } \left[n^{2} N_{1}^{2}\left(p-\mathrm{m}_{2}\right)+2 n N_{1}\left(N_{2}\left(\mathrm{~m}_{2}-3 p\right)+N_{1} p\right)\right.\right. \\
& \left.+4 N_{2}\left(N_{1} p+\left(2 p-\mathrm{m}_{2}\right) N_{2}\right)\right]-2 \mathrm{n}_{2} N_{1}\left(\mathrm{~m}_{2} n N_{1}+2 N_{2}\left(\mathrm{~m}_{2}-p\right)+(n-4) N_{1} p\right) \\
& \left.-4 \mathrm{n}_{2}^{2} N_{1}^{2}\right\} /\left\{N _ { 1 } ^ { 2 } \left[\mathrm{m}_{2}^{2} n^{2}-\mathrm{m}_{2} n(n+2) p+2 \mathrm{~m}_{2} n \mathrm{n}_{2}+(n-2)^{2} p^{2}+2(n-4) \mathrm{n}_{2} p\right.\right. \\
& \left.+4 \mathrm{n}_{2}^{2}\right]-2 N_{1} N_{2}\left(2 p-\mathrm{m}_{2}\right)\left((n-2) p-\mathrm{m}_{2} n\right)+4 \mathrm{n}_{2} N_{1} N_{2}\left(\mathrm{~m}_{2}-p\right) \\
& \left.+4 N_{2}^{2}\left(p-\mathrm{m}_{2}\right)^{2}\right\} .
\end{aligned}
$$

We may compare certain special cases of the rather unwieldy general result (5.21) with our earlier results. First, taking $n=0$ gives the product base $B_{4}=\mathbb{C} P^{1} \times \mathbb{C} P^{1}=S^{2} \times S^{2}$, which is the genus $g_{1}=0=g_{2}$ case from subsection 5.1.2. Further specializing to the symmetric case where we choose $\mathrm{n}_{2}=\mathrm{m}_{2} \equiv k, N_{1}=N_{2} \equiv N$, we find that (5.21) agrees precisely with the product of Riemann surfaces central charge (5.14) with $g_{1}=0=g_{2}$, as it should do. 
Secondly, $\mathbb{F}_{1}=d P_{1}$. Comparing to the notation of subsection 5.1.1, the north and south pole sections of $\mathbb{F}_{1}$ then have Poincaré duals $\hat{S}_{1}=H, \hat{S}_{2}=E_{1}$, where recall that $H$ is the hyperplane class and $E_{1}$ is the exceptional divisor class. The basis $\left\{e_{1}=\hat{F}, e_{2}=\hat{S}_{1}\right\}$ we have used in this subsection is hence $\left\{e_{1}=H-E_{1}, e_{2}=H\right\}$. Moreover, the quantum number $N$ in subsection 5.1.1 is by definition the flux through the Poincaré dual of the anti-canonical class $3 H-E_{1}=e_{1}+2 e_{2}$, implying that $N=N_{1}+2 N_{2}$. Moreover, since in (5.2) both $\left[J_{B_{4}}\right]$ and $\left[F_{i}\right]$ are proportional to the same class $c_{1}=3 H-E_{1}=e_{1}+2 e_{2}$, from the expression (4.23) for the flux quantum numbers $\left\{N_{1}, N_{2}\right\}$, defined to be the flux through the Poincaré duals to $\left\{e_{1}, e_{2}\right\}$, respectively, we deduce that

$$
\left(\begin{array}{l}
N_{1} \\
N_{2}
\end{array}\right) \propto I \cdot\left(\begin{array}{l}
1 \\
2
\end{array}\right)=\left(\begin{array}{l}
2 \\
3
\end{array}\right) .
$$

Thus $N_{2}=3 N_{1} / 2$. Combining this with $N=N_{1}+2 N_{2}$ above we deduce that $N_{1}=$ $N / 4, N_{2}=3 N / 8$. Finally, the U(1) flavour twisting in (5.2) satisfies $\frac{1}{2 \pi}\left[F_{2}\right]=n_{2} c_{1}$ and comparing with (5.17), (5.19) we can identify

$$
\mathrm{n}_{2}=(2-n) n_{2}=n_{2} \quad \mathrm{~m}_{2}=2 n_{2},
$$

where we set $n=1$ for the first Hirzebruch surface $\mathbb{F}_{1}$. Making these substitutions in (5.21) one finds

$$
c_{\text {sugra }}=\frac{9 n_{2} p\left(n_{2}-p\right)}{8\left(p^{2}-3 n_{2} p+3 n_{2}^{2}\right)} N^{2} .
$$

This agrees with the central charge (5.8) for $d P_{1}$ on setting $m_{1}=1, M^{(1)}=9-1=8$ for the first del Pezzo surface. Of course, in this case $d P_{1}$ does not admit a Kähler-Einstein metric, and so we cannot compare with the explicit supergravity solution result (D.2). However, it is natural to conjecture that a corresponding GK supergravity solution does exist in this case, but simply outside the Kähler-Einstein ansatz utilized in [17]. Similar remarks apply to the central charge (5.8) for second del Pezzo surface $k=2$, which is also not Kähler-Einstein. Whether GK supergravity solutions exist for general Hirzebruch surfaces $\mathbb{F}_{n}$, for which the central charge is then given by (5.21), is an interesting open problem.

\section{$5.2 \quad D=11$}

In this subsection we consider an $A d S_{2} \times Y_{9}$ example of the form $X_{3} \hookrightarrow Y_{9} \rightarrow B_{6}$, where we take the Kähler base to be $B_{6}=\mathbb{C} P^{3}$

We let $H$ generate the second cohomology $H=1 \in H^{2}\left(\mathbb{C} P^{3}, \mathbb{Z}\right) \cong \mathbb{Z}$ of $\mathbb{C} P^{3}$, which satisfies $\int_{\mathbb{C} P^{3}} H^{3}=1$. We may then write the cohomology classes of the Kähler form $J_{B_{6}}$ and curvature two-forms $F_{i}$ in $H^{2}\left(\mathbb{C} P^{3}, \mathbb{R}\right)$ as

$$
\left[J_{B_{6}}\right]=A H, \quad \frac{1}{2 \pi}\left[F_{i}\right]=n_{i} H,
$$

where $i=1,2$ and $A \in \mathbb{R}, n_{i} \in \mathbb{Z}$. Furthermore, from (4.12) we then have $n_{1}=4$. This case has three flux quantum numbers: $N, M_{i}$, for $i=1,2$. In carrying out our general procedure 
we will see that some ambiguities arise. We believe that it should be possible to fix these ambiguities by imposing suitable positivity conditions on the Kähler class parameters $A$ and $\lambda_{a}$, but we leave a general discussion of this for future work. Here we are content to show that there is a solution that gives precisely the same value for the entropy as that obtained from the explicit supergravity solutions discussed in appendix D.2.

We first solve the constraint equation (4.29) for $A$, finding two solutions. In continuing the procedure, we find that one of these solutions ultimately gives rise to an action function that, after setting $b_{1}=1$ as required for an $A d S_{2}$ solution, only depends linearly on $b_{2}$ and hence we cannot solve for $b_{2}$ after extremizing this action. We thus continue with the other solution for $A$ which, with $b_{1}=1$, and $n_{1}=4$, is given by

$$
A=\frac{2 \pi\left(4 b_{2}-n_{2}\right)\left[b_{2}^{2} \lambda_{2}\left(4 p-n_{2}\right)-\lambda_{1} n_{2}\left(b_{2}-p\right)^{2}\right]}{b_{2}\left(p-b_{2}\right)\left(-2 b_{2} n_{2}+4 b_{2} p+n_{2} p\right)} .
$$

We next solve the expression for the preferred flux $N$, given in (4.31), for $\lambda_{1}$, again finding two solutions. These are rather lengthy and we do not record them here. For both solutions the remaining two fluxes take the form

$$
M_{1}=\frac{\left(n_{2}-4 p\right)}{p} N, \quad M_{2}=-\frac{n_{2}}{p} N,
$$

which we note implies that $N$ is divisible by $p$. Furthermore, the two solutions for $\lambda_{1}$ just give rise to a change in sign of the action. We find that one of these solutions, which we now continue with, leads to precisely the entropy of the explicit solutions in appendix D.2. We then obtain an expression for the off-shell entropy function, and may set $b_{1}=1$. After varying with respect to the remaining $R$-symmetry direction $b_{2}$ we find that there are two extremal values for $b_{2}$, one of which connects with the explicit supergravity solutions. Assuming $p>0$ and $n_{2}<0$, which we will see in a moment are conditions imposed from the explicit solutions in appendix D.2, we find that this specific solution for $b_{2}$ is given by

$$
b_{2}=\frac{n_{2}\left(\sqrt{-4 n_{2} p+n_{2}^{2}+8 p^{2}}+n_{2}-4 p\right)}{4 \sqrt{-4 n_{2} p+n_{2}^{2}+8 p^{2}}} .
$$

Furthermore, with this value of $b_{2}$, the on-shell entropy $\mathscr{S}$, given by (2.17) and (4.36), takes the form

$$
\mathscr{S}=\frac{\sqrt{2} \pi\left(\sqrt{-4 n_{2} p+n_{2}^{2}+8 p^{2}}+n_{2}-2 p\right) \sqrt{n_{2}\left(n_{2}-4 p\right)}}{3 p^{3 / 2}} N^{3 / 2} .
$$

Having obtained this result from our general procedure, we may now compare with the explicit $A d S_{2} \times \mathscr{Y}^{\mathrm{p}, \mathrm{q}}\left(\mathrm{KE}_{6}^{+}\right)$solutions of $D=11$ supergravity that are discussed in appendix D.2. These solutions are labelled by two relatively prime integers $p, q>0$, as well as an overall flux number $n$. The parameters are related by $\mathrm{p}=-n_{2}, \mathbf{q}=p$ and $n=N / p$. Notice that these imply $p>0$ and $n_{2}<0$ which we used above, and also that the identification on $n$ is consistent with the fact that, as noted above, $p$ divides $N$. We then have that the fluxes of the $D=11$ solution given in (D.19) are related to $M_{1}, M_{2}$ via $N\left(D_{0}\right)=M_{1}, N\left(\tilde{D}_{0}\right)=-M_{2}$. Finally, one can also check that the on-shell entropy given in (5.29) precisely agrees with that given in (D.21). 


\section{Discussion}

We have studied the geometric extremal problem, introduced in [3], for GK manifolds $Y_{2 n+1}, n \geq 3$, that are toric fibrations over a Kähler base manifold $B_{2 k}$. Our results extend those of $[9,16]$, which studied the cases of $Y_{7}$ and $Y_{9}$ torically fibred over a Riemann surface $B_{2}=\Sigma_{g}$, respectively. Similar to $[9,16]$, we have shown that the relevant flux quantization conditions and the constraint condition, as well as the action function that determines the supersymmetric $R$-symmetry Killing vector, may all be written in terms of the master volume of the toric fibre, together with certain global data associated with the Kähler base. We have also checked our new formulae using explicit classes of supergravity solutions of the form $A d S_{3} \times Y_{7}$ and $A d S_{2} \times Y_{9}$, finding exact agreement.

When introducing the toric fibres our starting point was to consider them to be Sasaki. Such fibres have toric data, specified by a set of inward pointing normal vectors $\vec{v}_{a}$, that are associated with convex polyhedral cones. However, we know from explicit examples that this is too restrictive and we should also allow vectors $\vec{v}_{a}$ that are associated with "non-convex" toric cones, as introduced in [16]. Further study of such novel toric geometry is certainly warranted and this could also help to resolve the ambiguities in carrying out the extremal problem that we saw in section 5.2 for certain examples. More generally, an important outstanding topic is to determine the necessary and sufficient conditions for the existence of the GK geometries, given the Kähler base and the toric fibre data.

A natural way in which the $A d S_{3} \times Y_{7}$ and $A d S_{2} \times Y_{9}$ supergravity solutions arise is to consider wrapping a stack of D3-branes or membranes on a holomorphic curve in a Calabi-Yau four-fold or five-fold, respectively, as clarified in [20, 21]. These configurations give rise to supersymmetric field theories in the unwrapped directions of the branes, and when these flow to a conformal fixed point, the supergravity dual develops an $A d S_{3}$ or $A d S_{2}$ factor, respectively. This perspective should be helpful in further understanding the geometries we have studied in this paper, as well as identifying the dual SCFTs.

\section{Acknowledgments}

We would like to thank the Centro de Ciencias de Benasque Pedro Pascual for hospitality while part of this work was carried out. JPG is supported by the European Research Council under the European Union's Seventh Framework Programme (FP7/2007-2013), ERC Grant agreement ADG 339140. JPG is also supported by STFC grant ST/P000762/1, EPSRC grant EP/K034456/1, as a KIAS Scholar and as a Visiting Fellow at the Perimeter Institute. JPG acknowledges hospitality from the KITP and support from National Science Foundation under Grant No. NSF PHY-1748958. DM would like to thank the Galileo Galilei Institute for hospitality while this work was being carried out.

\section{A Master volume identities}

In this appendix we derive a number of identities satisfied by the master volume $\mathcal{V}$, that are used in the main text. 
As described in section 3.2 , we begin by introducing the new coordinates $x_{i} \equiv y_{i}-y_{i}^{(0)}$, $i=1, \ldots, r+1$, on $\mathbb{R}^{r+1}$, so that we may write the master volume as

$$
\mathcal{V}=(2 \pi)^{r+1} \int_{\mathbb{R}^{r+1}} \prod_{b=1}^{d} \theta\left(\left(\vec{x}, \vec{v}_{b}\right)-\lambda_{b}\right) \delta((\vec{x}, \vec{b}))
$$

Here the integration uses the standard Euclidean measure $\mathrm{d} x_{1} \wedge \cdots \wedge \mathrm{d} x_{r+1}$. Using Stokes' theorem we have

$$
\int_{\mathbb{R}^{r+1}} \nabla\left[f(\vec{x}) \prod_{b=1}^{d} \theta\left(\left(\vec{x}, \vec{v}_{b}\right)-\lambda_{b}\right) \delta((\vec{x}, \vec{b}))\right]=0,
$$

where $f(\vec{x})$ is an arbitrary function. The boundary term at infinity here vanishes on integrating by parts, because the term in square brackets is compactly supported (on a compact polytope embedded in $\mathbb{R}^{r+1}$ ). Taking $f \equiv 1$ to be constant, and then computing the gradient, one obtains

$$
\begin{aligned}
& \sum_{a=1}^{d} \vec{v}_{a} \int_{\mathbb{R}^{r+1}} \delta\left(\left(\vec{x}, \vec{v}_{a}\right)-\lambda_{a}\right) \prod_{b \neq a} \theta\left(\left(\vec{x}, \vec{v}_{b}\right)-\lambda_{b}\right) \delta((\vec{x}, \vec{b})) \\
& +\vec{b} \int_{\mathbb{R}^{r+1}} \prod_{b=1}^{d} \theta\left(\left(\vec{x}, \vec{v}_{b}\right)-\lambda_{b}\right) \delta^{\prime}((\vec{x}, \vec{b}))=0 .
\end{aligned}
$$

Note immediately that the integral on the first line is proportional to $\partial \mathcal{V} / \partial \lambda_{a}$. We next need to deal with the derivative of the $\delta$-function on the second line. Using the chain rule $\nabla=\vec{b} \partial_{s}$, where $s \equiv(\vec{x}, \vec{b})$ is the argument of the $\delta$-function, and it follows that we may write $\delta^{\prime}(s)=\frac{\vec{b} \cdot \nabla}{|\vec{b}|^{2}} \delta(s)$. We then integrate the second line of (A.3) by parts to obtain

$$
\sum_{a=1}^{d} \vec{v}_{a} \frac{\partial \mathcal{V}}{\partial \lambda_{a}}+(2 \pi)^{r+1} \vec{b} \sum_{a=1}^{d} \frac{\vec{b} \cdot \vec{v}_{a}}{|\vec{b}|^{2}} \int_{\mathbb{R}^{r+1}} \delta\left(\left(\vec{x}, \vec{v}_{a}\right)-\lambda_{a}\right) \prod_{b \neq a} \theta\left(\left(\vec{x}, \vec{v}_{b}\right)-\lambda_{b}\right) \delta((\vec{x}, \vec{b}))=0
$$

which immediately gives

$$
\sum_{a=1}^{d}\left(\vec{v}_{a}-\vec{b} \frac{\vec{b} \cdot \vec{v}_{a}}{|\vec{b}|^{2}}\right) \frac{\partial \mathcal{V}}{\partial \lambda_{a}}=0
$$

Notice this identity holds for arbitrary vectors $\left\{\vec{v}_{a}\right\}$ and $\vec{b}$. When $v_{a}^{1}=1$ for all $a=1, \ldots, d$, which is true when $C\left(X_{2 r+1}\right)$ is Gorenstein, one may take the $i=1$ component of (A.4), and substituting this back in one immediately derives

$$
\sum_{a=1}^{d}\left(\vec{v}_{a}-\frac{\vec{b}}{b_{1}}\right) \frac{\partial \mathcal{V}}{\partial \lambda_{a}}=0
$$

which is equation (3.20) in the main text. 
We next compute, from (A.1), that

$$
\frac{\partial \mathcal{V}}{\partial b_{i}}=(2 \pi)^{r+1} \int_{\mathbb{R}^{r+1}} \prod_{b=1}^{d} \theta\left(\left(\vec{x}, \vec{v}_{b}\right)-\lambda_{b}\right) \delta^{\prime}((\vec{x}, \vec{b})) x^{i} .
$$

Integrating the $\delta$-function by parts, as we did above, one finds

$$
\begin{aligned}
\frac{\partial \mathcal{V}}{\partial b_{i}}= & -(2 \pi)^{r+1} \sum_{a=1}^{d} \frac{\vec{b} \cdot \vec{v}_{a}}{|\vec{b}|^{2}} \int_{\mathbb{R}^{r+1}} \delta\left(\left(\vec{x}, \vec{v}_{a}\right)-\lambda_{a}\right) \prod_{b \neq a} \theta\left(\left(\vec{x}, \vec{v}_{b}\right)-\lambda_{b}\right) \delta((\vec{x}, \vec{b})) x^{i} \\
& -(2 \pi)^{r+1} \frac{b^{i}}{|\vec{b}|^{2}} \int_{\mathbb{R}^{r+1}} \prod_{b=1}^{d} \theta\left(\left(\vec{x}, \vec{v}_{b}\right)-\lambda_{b}\right) \delta((\vec{x}, \vec{b})) .
\end{aligned}
$$

But this simply reads

$$
\frac{\partial \mathcal{V}}{\partial b_{i}}=-\sum_{a=1}^{d} \frac{\vec{b} \cdot \vec{v}_{a}}{|\vec{b}|^{2}} \frac{\partial \mathcal{V}}{\partial v_{a}^{i}}-\frac{b^{i}}{|\vec{b}|^{2}} \mathcal{V} .
$$

Note that we can immediately deduce that

$$
\vec{b} \cdot \frac{\partial \mathcal{V}}{\partial \vec{v}_{a}}=0, \quad a=1, \ldots, d,
$$

by computing the expression for $\partial \mathcal{V} / \partial v_{a}^{i}$. Dotting (A.8) with $\vec{b}$ then implies that $\mathcal{V}$ is homogeneous degree -1 in $b_{i}$.

Next taking $f=x^{j}$ in (A.2) and computing in a similar way, we derive

$$
\delta_{i j} \mathcal{V}+\sum_{a=1}^{d} v_{a}^{i} \frac{\partial \mathcal{V}}{\partial v_{a}^{j}}-b_{i} \sum_{a=1}^{d} \frac{\vec{b} \cdot \vec{v}_{a}}{|\vec{b}|^{2}} \frac{\partial \mathcal{V}}{\partial v_{a}^{j}}-\frac{b_{i} b_{j}}{|\vec{b}|^{2}} \mathcal{V}=0
$$

Combining this with (A.8) then gives the remarkably simple identity

$$
\delta_{i j} \mathcal{V}+b_{i} \frac{\partial \mathcal{V}}{\partial b_{j}}+\sum_{a=1}^{d} v_{a}^{i} \frac{\partial \mathcal{V}}{\partial v_{a}^{j}}=0 .
$$

Contracting indices implies that for $X_{2 r+1}$, where recall $i, j=1, \ldots, r+1$, we have

$$
\sum_{a=1}^{d} \vec{v}_{a} \cdot \frac{\partial \mathcal{V}}{\partial \vec{v}_{a}}+r \mathcal{V}=0
$$

Differentiating (A.11) with respect to $b_{k}$ then immediately gives

$$
\delta_{i j} \frac{\partial \mathcal{V}}{\partial b_{k}}+\delta_{i k} \frac{\partial \mathcal{V}}{\partial b_{j}}+b_{i} \frac{\partial^{2} \mathcal{V}}{\partial b_{j} \partial b_{k}}+\sum_{a=1}^{d} v_{a}^{i} \frac{\partial^{2} \mathcal{V}}{\partial v_{a}^{j} \partial b_{k}}=0
$$

or equivalently

$$
\left[\frac{\partial \mathcal{V}}{\partial b_{j}} \delta_{k i}+\frac{b_{i}}{2} \frac{\partial^{2} \mathcal{V}}{\partial b_{j} \partial b_{k}}+\frac{1}{2} \sum_{a=1}^{d} v_{a}^{i} \frac{\partial^{2} \mathcal{V}}{\partial v_{a}^{j} \partial b_{k}}\right]_{\operatorname{Sym}(j, k)}=0
$$


where $\operatorname{Sym}(j, k)$ denotes that we should symmetrize over the $j, k$ indices. Equation (A.14) is another identity used in deriving results in the main text. Notice that we may compute

$$
\frac{\partial^{2} \mathcal{V}}{\partial v_{a}^{j} \partial b_{k}}=(2 \pi)^{r+1} \int_{\mathbb{R}^{r+1}} \delta\left(\left(\vec{x}, \vec{v}_{a}\right)-\lambda_{a}\right) \prod_{b \neq a} \theta\left(\left(\vec{x}, \vec{v}_{b}\right)-\lambda_{b}\right) \delta^{\prime}((\vec{x}, \vec{b})) x^{j} x^{k},
$$

where the right hand side is manifestly symmetric in $j$ and $k$. Finally, using (A.13) one easily derives the identity

$$
\begin{gathered}
{\left[\sum_{b=1}^{d} \lambda^{b} \frac{\partial}{\partial v_{b}^{l}}\left(\frac{1}{2} \frac{\partial \mathcal{V}}{\partial b_{j}} \delta_{k i}+\frac{b_{i}}{3 !} \frac{\partial^{2} \mathcal{V}}{\partial b_{j} \partial b_{k}}+\frac{1}{3 !} \sum_{a=1}^{d} v_{a}^{i} \frac{\partial^{2} \mathcal{V}}{\partial v^{a}{ }_{j} \partial b_{k}}\right)\right.} \\
\left.-\frac{1}{3 !} \sum_{b=1}^{d} \lambda^{b} \frac{\partial^{2} \mathcal{V}}{\partial v_{j}^{b} \partial b_{k}} \delta_{l i}\right]_{\operatorname{Sym}(j, k, l)}=0,
\end{gathered}
$$

where $\operatorname{Sym}(j, k, l)$ denotes that we should symmetrize over the $j, k, l$ indices. Again, this is another identity needed to obtain results in the main text.

\section{B Master volume for $\boldsymbol{X}_{3}$}

In this appendix we derive the formula (3.26) for the master volume of $X_{3}$.

As in section 3 we take the fibre $X_{3}$ to be the link of a Gorenstein Kähler cone of complex dimension $r+1=2$. The toric data is then $v_{1}=(1,0), v_{2}=(1, p), p \in \mathbb{N}$, which describes an $A_{p-1}$ singularity, $C\left(X_{3}\right)=\mathbb{C}^{2} / \mathbb{Z}_{p}$. Here the $\mathbb{Z}_{p}$ action on $\mathbb{C}^{2}$ is $\left(z_{1}, z_{2}\right) \mapsto$ $\left(\omega_{p} z_{1}, \omega_{p}^{-1} z_{2}\right)$, where $\omega_{p}$ is a primitive $p$ th root of unity. The outward pointing normals to the edges at the apex are $u_{1}=(0,1), u_{2}=(p,-1)$. Recall also that the "origin" of the polytope $\mathcal{P}$ is located at

$$
\vec{y}^{(0)}=\left(\frac{1}{2 b_{1}}, 0\right)
$$

Denoting by $\lambda_{1}, \lambda_{2}$ the Kähler parameters associated to the two facets, as in the main text, then the two vertices $\vec{y}_{a}, a=1,2$, of the polytope $\mathcal{P}$, which here is a line segment, satisfy the equations

$$
\left(\vec{y}_{a}-\vec{y}^{(0)}, \vec{b}\right)=0, \quad\left(\vec{y}_{a}-\vec{y}^{(0)}, \vec{v}_{a}\right)=\lambda_{a}, \quad a=1,2
$$

These are easily solved to give

$$
\left(\vec{y}_{a}-\vec{y}^{(0)}\right)_{i}=\frac{\lambda_{a} \varepsilon_{i j} b^{j}}{\varepsilon_{m n} v_{a}^{m} b^{n}}
$$

The master volume (3.15) is

$$
\mathcal{V}=\frac{(2 \pi)^{2}}{|\vec{b}|} \operatorname{vol}(\mathcal{P})
$$


where here the "volume" of the polytope $\mathcal{P}$ is simply the length of the line segment between the two vertices in (B.4). But this is

$$
\operatorname{vol}(\mathcal{P})=\left(-\frac{\lambda_{1} \varepsilon_{i j} b^{j}}{\varepsilon_{m n} v_{1}^{m} b^{n}}+\frac{\lambda_{2} \varepsilon_{i j} b^{j}}{\varepsilon_{m n} v_{2}^{m} b^{n}}\right) \frac{\varepsilon_{i k} b^{k}}{|\vec{b}|} .
$$

Here each of the two terms in the bracket is the vector from the origin to the corresponding vertex. To compute the length we have then taken a two-dimensional cross product with the unit normal $\vec{b} /|\vec{b}|$. A short computation then gives the simple formula

$$
\mathcal{V}=(2 \pi)^{2} \sum_{a=1}^{2}(-1)^{a} \frac{\lambda_{a}}{\left[\vec{v}_{a}, \vec{b}\right]}=(2 \pi)^{2}\left(-\frac{\lambda_{1}}{b_{2}}+\frac{\lambda_{2}}{b_{2}-b_{1} p}\right),
$$

where here $\left[\vec{v}_{a}, \vec{b}\right]$ is the determinant of the $2 \times 2$ matrix, i.e. $\left[\vec{v}_{a}, \vec{b}\right] \equiv \varepsilon_{m n} v_{a}^{m} b^{n}$.

Notice that setting $\lambda_{a}=-\frac{1}{2 b_{1}}$ for $a=1,2$, and then $b_{1}=2$, the extremum of the corresponding volume function $\mathcal{V}$ occurs at $b_{2}=p$, with extremal volume $\mathcal{V}=2 \pi^{2} / p$. This is the correct volume of the Lens space $L(1, p)=S^{3} / \mathbb{Z}_{p}$, equipped with its Sasaki-Einstein metric.

\section{More on the master volume}

In section 4 we presented formulae for the constraint (2.8), flux quantization conditions (2.9) and supersymmetric action (2.10) for fibred GK geometries, where crucially the formulas depend only on certain topological integrals over the Kähler base $B_{2 k}$, together with derivatives of the master volume $\mathcal{V}$ of the fibres $X_{2 r+1}$. Here $\mathcal{V}=\mathcal{V}\left(\vec{b} ;\left\{\lambda_{a}\right\} ;\left\{\vec{v}_{a}\right\}\right)$ is a function of the $R$-symmetry vector $\vec{b}$, Kähler class parameters $\left\{\lambda_{a}\right\}$, and the toric data $\left\{\vec{v}_{a}\right\}$ of the cone $C\left(X_{2 r+1}\right)$. Derivatives of $\mathcal{V}$ with respect to all three appear. In section C.1 we derive various formulae for these derivatives that we have used in deriving the results of sections 4.2, 4.3 and 4.4. In section C.2 we explicitly explain how these have been used to derive the expressions in section 4.3 - the results of sections 4.2 and 4.4 are obtained similarly. As we have seen, there are various equivalent ways to write the master volume, and some forms are more useful than others in deriving particular formulae. We have already presented a number of identities satisfied by the master volume and its derivatives in appendix A.

\section{C.1 Derivatives of the master volume}

The master volume of the fibre $X_{2 r+1}$ can be written variously as

$$
\begin{aligned}
\mathcal{V} & =\int_{X_{2 r+1}} \eta \wedge \frac{1}{r !} \omega^{r} \\
& =\frac{(2 \pi)^{r+1}}{|\vec{b}|} \operatorname{vol}(\mathcal{P}) \\
& =(2 \pi)^{r+1} \int_{\mathbb{R}^{r+1}} \prod_{a=1}^{d} \theta\left(\left(\vec{x}, \vec{v}_{a}\right)-\lambda_{a}\right) \delta((\vec{x}, \vec{b})) .
\end{aligned}
$$


The first equality is the original definition (3.11), while the second writes this in terms of the Euclidean volume of the moment map polytope $\mathcal{P}$, where the latter is defined in equation (3.16), while the third equality writes this Euclidean volume in terms of step functions and a $\delta$-function integrated over $\mathbb{R}^{r+1}$. Recall here that $\mathbb{R}^{r+1}$ is parametrized by the shifted moment map variables $x_{i}, i=1, \ldots, r+1$, defined in (3.18), where we have suppressed the standard Euclidean measure $\mathrm{d} x_{1} \wedge \cdots \wedge \mathrm{d} x_{r+1}$ in the notation, to keep formulae uncluttered.

Using equation (3.25) with $s=0$ we also have

$$
\begin{aligned}
-\frac{1}{2 \pi} \frac{\partial \mathcal{V}}{\partial \lambda_{a}} & =\int_{S_{a}} \eta \wedge \frac{1}{(r-1) !} \omega^{r-1} \\
& =(2 \pi)^{r} \int_{\mathbb{R}^{r+1}} \delta\left(\left(\vec{x}, \vec{v}_{a}\right)-\lambda_{a}\right) \prod_{b \neq a} \theta\left(\left(\vec{x}, \vec{v}_{b}\right)-\lambda_{b}\right) \delta((\vec{x}, \vec{b})) .
\end{aligned}
$$

Recall here that $S_{a} \subset X_{2 r+1}$ is the $(2 r-1)$-submanifold in $X_{2 r+1}$ associated with the $a$ th toric divisor on the cone $C\left(X_{2 r+1}\right)$, which moreover is Poincaré dual to $c_{a}$. Here the second line of (C.2) follows immediately by differentiating the expression in the third line of (C.1) with respect to $\lambda_{a}$.

Starting with the third line of (C.1), we compute ${ }^{6}$

$$
\begin{aligned}
\frac{\partial \mathcal{V}}{\partial v_{a}^{i}} & =(2 \pi)^{r+1} \int_{\mathbb{R}^{r+1}} \delta\left(\left(\vec{x}, \vec{v}_{a}\right)-\lambda_{a}\right) \prod_{b \neq a} \theta\left(\left(\vec{x}, \vec{v}_{b}\right)-\lambda_{b}\right) \delta((\vec{x}, \vec{b})) x_{i} \\
& =(2 \pi) \int_{S_{a}} x_{i} \eta \wedge \frac{1}{(r-1) !} \omega^{r-1} .
\end{aligned}
$$

In the second equality we have rewritten the expression as an integral over $S_{a}$, as in (C.2), where in doing so notice that $x_{i}$ may be interpreted as a Hamiltonian function for the transverse Kähler two-form $\omega$, as introduced in (4.9). Using the first line of (C.3) we also have

$$
\begin{aligned}
\sum_{a=1}^{d} \lambda^{a} \frac{\partial \mathcal{V}}{\partial v_{a}^{i}} & =-\sum_{a=1}^{d} \lambda^{a} \frac{\partial}{\partial \lambda^{a}}\left[(2 \pi)^{r+1} \int_{\mathbb{R}^{r+1}} \prod_{b=1}^{d} \theta\left(\left(\vec{x}, \vec{v}_{b}\right)-\lambda_{b}\right) \delta((\vec{x}, \vec{b})) x_{i}\right] \\
& =-(r+1) \int_{X_{2 r+1}} x_{i} \eta \wedge \frac{1}{r !} \omega^{r}
\end{aligned}
$$

where the second equality comes from the fact that the quantity in square brackets is homogeneous degree $r+1$ in the $\lambda_{a}$. More generally, we may similarly deduce

$$
\sum_{a_{1}, \ldots, a_{s}=1}^{d} \lambda^{a_{1}} \ldots \lambda^{a_{s}} \frac{\partial^{s} \mathcal{V}}{\partial v_{a_{1}}^{i_{1}} \ldots \partial v_{a_{s}}^{i_{s}}}=(-1)^{s}\left[\prod_{m=1}^{s}(r+m)\right] \int_{X_{2 r+1}} x_{i_{1}} \ldots x_{i_{s}} \eta \wedge \frac{1}{r !} \omega^{r} .
$$

\footnotetext{
${ }^{6}$ It is perhaps worth noting that one cannot use Poincaré duality to replace the integral over $S_{a}$ in the expression on the second line of (C.3) as an integral over $X_{2 r+1}$, simply because the integrand is not a closed form.
} 
From this, and similarly to (C.3), we also deduce that

$$
\begin{aligned}
& \sum_{a_{1}, \ldots, a_{s}=1}^{d} \lambda^{a_{1}} \ldots \lambda^{a_{s}} \frac{\partial^{s+1} \mathcal{V}}{\partial v_{a_{1}}^{i_{1}} \ldots \partial v_{a_{s}}^{i_{s}} \partial v_{a}^{i}} \\
& \quad=(2 \pi)(-1)^{s}\left[\prod_{m=1}^{s}(r+m)\right] \int_{S_{a}} x_{i_{1}} \ldots x_{i_{s}} x_{i} \eta \wedge \frac{1}{(r-1) !} \omega^{r-1} .
\end{aligned}
$$

Next we would like to obtain expressions for derivatives of $\mathcal{V}$ with respect to the $R$ symmetry vector $\vec{b}$. These are obtained somewhat differently from the method above. First recall from section 4.1 that

$$
\left.\left.\left.\partial_{\varphi_{i}}\right\lrcorner \eta=2 w_{i}, \quad \partial_{\varphi_{i}}\right\lrcorner \mathrm{~d} \eta=-2 \mathrm{~d} w_{i}, \quad \partial_{\varphi_{i}}\right\lrcorner \omega=-\mathrm{d} x_{i} .
$$

We may differentiate $\mathcal{V}$, defined by the first equality in (C.1), with respect to the $R$ symmetry vector by taking the derivative inside the integral, and computing the corresponding first order variations of $\eta$ and $\omega$. Using the fact that the $R$-symmetry vector is

$$
\xi=\sum_{i=1}^{r+1} b_{i} \partial_{\varphi_{i}},
$$

together with $\xi\lrcorner \eta=1, \xi\lrcorner \omega=0$ and (C.7), following section 4.2 of [16] one finds the first order variations

$$
\begin{aligned}
\delta_{b_{j}} \eta & =-2 w_{j} \eta+\nu_{j}^{T}, \\
\delta_{b_{j}} \omega & =\eta \wedge \mathrm{d} x_{j}-x_{j} \mathrm{~d} \eta+\mathrm{d} \gamma_{j}^{T},
\end{aligned}
$$

where $\nu_{j}^{T}$ and $\gamma_{j}^{T}$ are basic forms for the foliation $\mathcal{F}_{\xi}$. Here in the variation of $\omega$ we hold the transverse Kähler class fixed, as in section 4.2 of [16]. In computing second derivatives of $\mathcal{V}$ we will also need the first order variations of $x_{i}$ and $w_{i}$. From (C.7) and (C.9) we may immediately deduce

$$
\left.\delta_{b_{j}} w_{i}=-2 w_{i} w_{j}+\frac{1}{2} \partial_{\varphi_{i}}\right\lrcorner \nu_{j}^{T} .
$$

Using the fact that the Lie derivatives of $x_{j}$ and $\gamma_{j}$ with respect to $\partial_{\varphi_{i}}$ vanish, we similarly find

$$
\left.\delta_{b_{j}} x_{i}=-2 w_{i} x_{j}+\partial_{\varphi_{i}}\right\lrcorner \gamma_{j}^{T} .
$$

With these results to hand, using the first definition of $\mathcal{V}$ in (C.1) it is straightforward to compute

$$
\begin{aligned}
\frac{\partial \mathcal{V}}{\partial b_{i}} & =-\int_{X_{2 r+1}} \eta \wedge\left[2 w_{i} \frac{\omega^{r}}{r !}+x_{i} \frac{\omega^{r-1}}{(r-1) !} \wedge \mathrm{d} \eta\right] \\
\frac{\partial^{2} \mathcal{V}}{\partial b_{i} \partial b_{j}} & =\int_{X_{2 r+1}} \eta \wedge\left[8 w_{i} w_{j} \frac{\omega^{r}}{r !}+8 w_{(i} x_{j} \frac{\omega^{r-1}}{(r-1) !} \wedge \mathrm{d} \eta+x_{i} x_{j} \frac{\omega^{r-2}}{(r-2) !} \wedge(\mathrm{d} \eta)^{2}\right] .
\end{aligned}
$$


We may similarly take the derivative of the expression for $\sum_{a=1}^{d} \partial \mathcal{V} / \partial \lambda_{a}$ given by (3.24) (with $s=1$ ) to obtain

$$
\begin{gathered}
\sum_{a=1}^{d} \frac{\partial^{2} \mathcal{V}}{\partial \lambda_{a} \partial b_{i}}=b_{1} \int_{X_{2 r+1}} \eta \wedge\left[4 w_{i} \frac{\omega^{r-1}}{(r-1) !} \wedge \mathrm{d} \eta+x_{i} \frac{\omega^{r-2}}{(r-2) !} \wedge(\mathrm{d} \eta)^{2}\right] \\
+\delta_{i 1} \frac{1}{b_{1}} \sum_{a=1}^{d} \frac{\partial \mathcal{V}}{\partial \lambda_{a}},
\end{gathered}
$$

where we have used $[\rho]=b_{1}[\mathrm{~d} \eta] \in H_{B}^{2}\left(\mathcal{F}_{\xi}\right)$ from (3.6). We shall also need

$$
-\frac{\partial}{\partial b_{j}} \int_{X_{2 r+1}} \eta \wedge \frac{1}{r !} \omega^{r} x_{i}=\int_{X_{2 r+1}} \eta \wedge\left[4 x_{(i} w_{j)} \frac{1}{r !} \omega^{r}+x_{i} x_{j} \frac{1}{(r-1) !} \omega^{r-1} \wedge \mathrm{d} \eta\right],
$$

and taking another derivative

$$
\begin{aligned}
\frac{\partial^{2}}{\partial b_{j} \partial b_{k}} \int_{X_{2 r+1}} \eta \wedge \frac{1}{r !} \omega^{r} x_{i}=\int_{X_{2 r+1}} \eta & \wedge\left[24 w_{(i} w_{j} x_{k)} \frac{1}{r !} \omega^{r}+12 w_{(i} x_{j} x_{k)} \frac{1}{(r-1) !} \omega^{r-1} \wedge \mathrm{d} \eta\right. \\
& \left.+x_{i} x_{j} x_{k} \frac{1}{(r-2) !} \omega^{r-2} \wedge(\mathrm{d} \eta)^{2}\right]
\end{aligned}
$$

Finally, we also have

$$
\begin{aligned}
-\frac{\partial}{\partial b_{k}} \int_{X_{2 r+1}} \eta \wedge \frac{1}{r !} \omega^{r} x_{i} x_{j}=\int_{Y_{2 r+1}} \eta & \wedge\left[6 w_{(i} x_{j} x_{k}\right) \frac{1}{r !} \omega^{r} \\
& \left.+x_{i} x_{j} x_{k} \frac{1}{(r-1) !} \omega^{r-1} \wedge \mathrm{d} \eta\right] .
\end{aligned}
$$

Turning now to integrals over the toric codimension two submanifolds $S_{a} \subset X_{2 r+1}$, taking the derivative of the expression given in (3.25) we find

$$
\begin{aligned}
\frac{(-1)^{s}}{2 \pi} \sum_{b_{1}, \ldots, b_{s}=1}^{d} \frac{\partial^{s+2} \mathcal{V}}{\partial b_{i} \partial \lambda_{a} \partial \lambda_{b_{1}} \ldots \partial \lambda_{b_{s}}}=\int_{S_{a}} \eta & \wedge \rho^{s} \wedge\left[2(1+s) w_{i} \frac{1}{(r-s-1) !} \omega^{r-s-1}\right. \\
& \left.+x_{i} \mathrm{~d} \eta \wedge \frac{1}{(r-s-2) !} \omega^{r-s-2}\right] .
\end{aligned}
$$

In particular for $s=0$ we have

$$
\frac{1}{2 \pi} \frac{\partial^{2} \mathcal{V}}{\partial b_{i} \partial \lambda_{a}}=\int_{S_{a}} \eta \wedge\left[2 w_{i} \frac{1}{(r-1) !} \omega^{r-1}+x_{i} \mathrm{~d} \eta \wedge \frac{1}{(r-2) !} \omega^{r-2}\right] .
$$

We similarly have

$$
\begin{aligned}
-\frac{\partial}{\partial b_{j}} \int_{S_{a}} \eta \wedge \frac{1}{(r-1) !} \omega^{r-1} x_{i}=\int_{S_{a}} \eta \wedge & {\left[4 w_{(i} x_{j} \frac{1}{(r-1) !} \omega^{r-1}\right.} \\
& \left.+x_{i} x_{j} \mathrm{~d} \eta \wedge \frac{1}{(r-2) !} \omega^{r-2}\right],
\end{aligned}
$$


as well as

$$
\begin{aligned}
-\frac{\partial}{\partial b_{k}} \int_{S_{a}} \eta \wedge \frac{1}{(r-1) !} \omega^{r-1} x_{i} x_{j}=\int_{S_{a}} \eta \wedge & {\left[6 w_{(i} x_{j} x_{k)} \frac{1}{(r-1) !} \omega^{r-1}\right.} \\
+ & \left.+x_{i} x_{j} x_{k} \mathrm{~d} \eta \wedge \frac{1}{(r-2) !} \omega^{r-2}\right] .
\end{aligned}
$$

\section{C.2 Formulae for $X_{2 r+1} \hookrightarrow Y_{2 r+5} \rightarrow B_{4}$}

In this subsection we explain how to derive the formulae presented in section 4.3, starting from the general expressions for the constraint (2.8), flux quantization conditions (2.9) and supersymmetric action (2.10).

Starting with the supersymmetric action (2.10), making the substitutions in (4.11) and recalling (3.6) immediately gives

$$
\begin{aligned}
S_{\mathrm{SUSY}}= & \int_{Y_{2 n+1}} \eta \wedge b_{1}\left(\mathrm{~d} \eta+2 w_{i} F_{i}\right) \wedge \frac{1}{(n-1) !}\left(\omega+x_{j} F_{j}+J_{B_{4}}\right)^{n-1} \\
= & \int_{Y_{2 n+1}} \eta \wedge b_{1} \mathrm{~d} \eta \wedge \frac{1}{(n-3) !} \omega^{n-3} \wedge \frac{1}{2} J_{B_{4}} \wedge J_{B_{4}} \\
& +b_{1} \int_{Y_{2 n+1}} \eta \wedge\left[2 w_{i} \frac{1}{(n-2) !} \omega^{n-2}+x_{i} \mathrm{~d} \eta \wedge \frac{1}{(n-3) !} \omega^{n-3}\right] \wedge F_{i} \wedge J_{B_{4}} \\
& +b_{1} \int_{Y_{2 n+1}} \eta \wedge\left[4 w_{(i} x_{j} \frac{1}{(n-2) !} \omega^{n-2}+x_{i} x_{j} \mathrm{~d} \eta \wedge \frac{1}{(n-3) !} \omega^{n-3}\right] \wedge \frac{1}{2} F_{i} \wedge F_{j} .
\end{aligned}
$$

Here in the second equality we have simply collected terms together and written them as a $(2 r+1)$-form on the fibre $X_{2 r+1}$ wedged with a four-form on the base $B_{4}$, where $n=r+2$. This then leads to the elegant expression

$$
\begin{aligned}
S_{\mathrm{SUSY}}=- & \sum_{a=1}^{d} \frac{\partial \mathcal{V}}{\partial \lambda_{a}} \operatorname{vol}\left(B_{4}\right)-b_{1} \sum_{i=1}^{r+1} \frac{\partial \mathcal{V}}{\partial b_{i}} \int_{B_{4}} F_{i} \wedge J_{B_{4}} \\
& +\frac{b_{1}}{r+1} \sum_{a=1}^{d} \sum_{i, j=1}^{r+1} \lambda_{a} \frac{\partial^{2} \mathcal{V}}{\partial b_{j} \partial v_{a}^{i}} \int_{B_{4}} \frac{1}{2} F_{i} \wedge F_{j},
\end{aligned}
$$

where $\operatorname{vol}\left(B_{4}\right)=\int_{B_{4}} \frac{1}{2} J_{B_{4}} \wedge J_{B_{4}}$. Here we have used equation (3.24) (with $s=1$ ) for the first term, equation (C.12) for the second term, and equation (C.14) for the third term. The final form of the third term presented in (C.22) then further uses the expression (C.4) for the left hand side of (C.14).

Next we turn to the constraint equation (2.8). Using (4.11) and (3.6) this similarly expands as

$$
\begin{aligned}
0= & \int_{Y_{2 n+1}} \eta \wedge b_{1}\left(\mathrm{~d} \eta+2 w_{i} F_{i}\right) \wedge b_{1}\left(\mathrm{~d} \eta+2 w_{j} F_{j}\right) \wedge \frac{1}{(n-2) !}\left(\omega+x_{k} F_{k}+J_{B_{4}}\right)^{n-2} \\
= & \int_{Y_{2 n+1}} \eta \wedge \rho^{2} \wedge \frac{1}{(n-4) !} \omega^{n-4} \wedge \frac{1}{2} J_{B_{4}} \wedge J_{B_{4}} \\
& +b_{1}^{2} \int_{Y_{2 n+1}} \eta \wedge\left[x_{i}(\mathrm{~d} \eta)^{2} \wedge \frac{1}{(n-4) !} \omega^{n-4}+4 w_{i} \mathrm{~d} \eta \wedge \frac{1}{(n-3) !} \omega^{n-3}\right] \wedge F_{i} \wedge J_{B_{4}}
\end{aligned}
$$




$$
\begin{aligned}
+b_{1}^{2} \int_{Y_{2 n+1}} \eta \wedge\left[8 w_{i} w_{j} \frac{1}{(n-2) !} \omega^{n-2}+8 w_{(i} x_{j} \mathrm{~d} \eta \wedge \frac{1}{(n-3) !} \omega^{n-3}\right. \\
\left.+x_{i} x_{j}(\mathrm{~d} \eta)^{2} \wedge \frac{1}{(n-4) !} \omega^{n-4}\right] \wedge \frac{1}{2} F_{i} \wedge F_{j},
\end{aligned}
$$

where again $n=r+2$. We may then use equation (3.24) (with $s=2$ ) for the first term, equation (C.13) for the second term, and equation (C.12) for the third term. This immediately gives

$$
\begin{aligned}
& \sum_{a, b=1}^{d} \frac{\partial^{2} \mathcal{V}}{\partial \lambda_{a} \partial \lambda_{b}} \operatorname{vol}\left(B_{4}\right)+b_{1} \sum_{i=1}^{r+1} \sum_{a=1}^{d} \frac{\partial^{2} \mathcal{V}}{\partial \lambda_{a} \partial b_{i}} \int_{B_{4}} F_{i} \wedge J_{B_{4}}-\sum_{a=1}^{d} \frac{\partial \mathcal{V}}{\partial \lambda_{a}} \int_{B_{4}} F_{1} \wedge J_{B_{4}} \\
& \quad+b_{1}^{2} \sum_{i, j=1}^{r+1} \frac{\partial^{2} \mathcal{V}}{\partial b_{i} \partial b_{j}} \int_{B_{4}} \frac{1}{2} F_{i} \wedge F_{j}=0
\end{aligned}
$$

which is the constraint equation (4.21) presented in the main text.

For the flux quantization condition there are two types of cycle. The first type has the fibred form $X_{2 r+1} \hookrightarrow \Sigma_{\alpha} \rightarrow C_{\alpha}^{(2)}$, where $C_{\alpha}^{(2)} \subset B_{4}$ is a two-cycle. In this case the flux quantization condition (2.9) reads

$$
\begin{aligned}
\nu_{n} N_{\alpha}= & \int_{\Sigma_{\alpha}} \eta \wedge b_{1}\left(\mathrm{~d} \eta+2 w_{i} F_{i}\right) \wedge \frac{1}{(n-2) !}\left(\omega+x_{j} F_{j}+J_{B_{4}}\right)^{n-2} \\
= & \int_{\Sigma_{\alpha}} \eta \wedge b_{1} \mathrm{~d} \eta \wedge \frac{1}{(n-3) !} \omega^{n-3} \wedge J_{B_{4}} \\
& +b_{1} \int_{\Sigma_{\alpha}} \eta \wedge\left[2 w_{i} \frac{1}{(n-2) !} \omega^{n-2} x_{i} \mathrm{~d} \eta \wedge \frac{1}{(n-3) !} \omega^{n-3}\right] \wedge F_{i} .
\end{aligned}
$$

Using equation (3.24) (with $s=1$ ) for the first term, and equation (C.12) for the second term then leads to

$$
\nu_{n} N_{\alpha}=-\sum_{a=1}^{d} \frac{\partial \mathcal{V}}{\partial \lambda_{a}} \int_{C_{\alpha}^{(2)}} J_{B_{4}}-b_{1} \sum_{i=1}^{r+1} \frac{\partial \mathcal{V}}{\partial b_{i}} \int_{C_{\alpha}^{(2)}} F_{i},
$$

which is equation (4.23) in the main text. The second set of cycles have the fibred form $S_{a} \hookrightarrow \Sigma_{a} \rightarrow B_{4}$, where $S_{a} \subset X_{2 r+1}$ is a toric codimension two submanifold in the fibre. In this case the flux quantization condition (2.9) reads

$$
\begin{aligned}
\nu_{n} M_{a}= & \int_{\Sigma_{a}} \eta \wedge b_{1}\left(\mathrm{~d} \eta+2 w_{i} F_{i}\right) \wedge \frac{1}{(n-2) !}\left(\omega+x_{j} F_{j}+J_{B_{4}}\right)^{n-2} \\
= & b_{1} \int_{\Sigma_{a}} \eta \wedge \mathrm{d} \eta \wedge \frac{1}{(n-4) !} \omega^{n-4} \wedge \frac{1}{2} J_{B_{4}} \wedge J_{B_{4}} \\
& +b_{1} \int_{\Sigma_{a}} \eta \wedge\left[x_{i} \mathrm{~d} \eta \wedge \frac{1}{(n-4) !} \omega^{n-4}+2 w_{i} \frac{1}{(n-3) !} \omega^{n-3}\right] \wedge F_{i} \wedge J_{B_{4}} \\
& +b_{1} \int_{\Sigma_{a}} \eta \wedge\left[x_{i} x_{j} \mathrm{~d} \eta \wedge \frac{1}{(n-4) !} \omega^{n-4}+4 w_{(i} x_{j} \frac{1}{(n-3) !} \omega^{n-3}\right] \wedge \frac{1}{2} F_{i} \wedge F_{j},
\end{aligned}
$$


where the flux quantum number is denoted $M_{a} \in \mathbb{Z}$. Using equation (3.25) (with $s=1$ ) for the first term, equation (C.18) for the second term, and combining equations (C.3) and (C.19) for the third term then leads to

$$
\begin{gathered}
\nu_{n} M_{a}=\frac{1}{2 \pi} \sum_{b=1}^{d} \frac{\partial^{2} \mathcal{V}}{\partial \lambda_{a} \partial \lambda_{b}} \operatorname{vol}\left(B_{4}\right)+\frac{b_{1}}{2 \pi} \sum_{i=1}^{r+1} \frac{\partial^{2} \mathcal{V}}{\partial \lambda_{a} \partial b_{i}} \int_{B_{4}} F_{i} \wedge J_{B_{4}} \\
-\frac{b_{1}}{2 \pi} \sum_{i, j=1}^{r+1} \frac{\partial^{2} \mathcal{V}}{\partial b_{j} \partial v_{a}^{i}} \int_{B_{4}} \frac{1}{2} F_{i} \wedge F_{j}
\end{gathered}
$$

which is equation (4.25) in the main text.

Finally, the form of the supersymmetric action presented in (4.28) may be obtained from (C.22) by first summing (C.28) over $a=1, \ldots, d$, and then using (C.26) together with the fact that the master volume $\mathcal{V}$ is homogeneous degree $r$ in the $\lambda_{a}$. The formulae presented in sections 4.2 and 4.4 are obtained in an entirely analogous manner, in particular using the equations we have so far not used in appendix C.1.

\section{Explicit solutions with Kähler-Einstein factors}

In this appendix we present some explicit solutions of type IIB and $D=11$ supergravity where the Kähler base $B_{2 k}$ is Kähler-Einstein with positive curvature. The results from this section are compared with some of the results obtained using our general formalism in section 5 .

\section{D.1 Type IIB: $\mathscr{Y}^{\mathrm{p}, \mathrm{q}}\left(\mathrm{KE}_{4}^{+}\right)$}

We first recall the class of explicit $A d S_{3} \times Y_{7}$ solutions of type IIB supergravity of the form (2.11) that were constructed in [17]. The solutions, which we label $Y_{7}=\mathscr{Y}^{\mathrm{p}, \mathrm{q}}\left(\mathrm{KE}_{4}^{+}\right)$, are constructed using an arbitrary Kähler-Einstein manifold with positive curvature, $\mathrm{KE}_{4}^{+}$, and are specified by two positive, relatively prime ${ }^{7}$ integers, $p, q>0$. As explained in detail in [18], $\mathscr{Y}^{\mathrm{p}, \mathrm{q}}\left(\mathrm{KE}_{4}^{+}\right)$can be constructed as a circle fibration over a regular six-dimensional manifold, which itself is obtained by constructing an $S^{2}$ bundle over $\mathrm{KE}_{4}^{+}$. Equivalently, $\mathscr{Y}^{\mathrm{p}, \mathrm{q}}\left(\mathrm{KE}_{4}^{+}\right)$can also be viewed as the total space of a Lens space $L(\mathrm{q}, 1)=S^{3} / \mathbb{Z}_{\mathrm{q}}$ fibred over $\mathrm{KE}_{4}^{+}$.

The analysis of the regularity of the solutions, flux quantization, and calculation of the central charge was carried out in detail in [17]. In the notation of [17] the flux integrals are given by

$$
\begin{aligned}
& N\left(D_{0}\right)=-\frac{M}{h m^{2}}(\mathrm{p}+m \mathrm{q}) n, \\
& N\left(\tilde{D}_{0}\right)=-\frac{M}{h m^{2}} \mathrm{p} n \\
& N\left(D_{a}\right)=\frac{\mathrm{q}}{h} n_{a} n
\end{aligned}
$$

\footnotetext{
${ }^{7}$ This ensures $Y_{7}$ is simply connected.
} 
while the central charge is given by

$$
c=\frac{9 \mathrm{pq}^{3}(\mathrm{p}+m \mathrm{q})}{3 \mathrm{p}^{2}+3 m \mathrm{pq}+m^{2} \mathrm{q}^{2}} \frac{M}{m^{2} h^{2}} n^{2},
$$

where $n$ is an arbitrary integer. In addition, the integers $m$ and $M$ depend on the specific choice of $\mathrm{KE}_{4}^{+}$. If $\mathcal{K}$ is the canonical line bundle of the $\mathrm{KE}_{4}^{+}$then the Fano index $m$ is the largest positive integer $m$ for which there is a line bundle $\mathcal{N}$ with $\mathcal{K}=\mathcal{N}^{m}$. If $\rho_{\text {KE }}$ is the Ricci-form of the $\mathrm{KE}_{4}^{+}$then $M=\int_{\mathrm{KE}_{4}^{+}}\left(\frac{1}{2 \pi} \rho_{\mathrm{KE}}\right)^{2}=\int_{\mathrm{KE}_{4}^{+}} c_{1}^{2}$. For $S^{2} \times S^{2}$ we then have $m=2, M=8$; for $\mathbb{C} P^{2}$ we have $m=3, M=9$; and for the del Pezzo surfaces $d P_{k}$, we have $m=1, M=9-k$, where $k=3, \ldots, 8$. Finally, $h=\operatorname{hcf}\left(M / m^{2}, \mathrm{q}\right)$.

\section{D.2 $D=11: \mathscr{Y}^{\mathrm{p}, \mathrm{q}}\left(\mathrm{KE}_{6}^{+}\right)$}

We next discuss a class of explicit $A d S_{2} \times Y_{9}$ solutions of $D=11$ supergravity of the form (2.15), with $Y_{9}=\mathscr{Y}^{\mathrm{p}, \mathrm{q}}\left(\mathrm{KE}_{6}^{+}\right)$. These solutions were first discussed in section 3.2 of [18], including determining the conditions required to obtain regular solutions, which are labelled by two positive, relatively prime integers, $p, q>0$. Here we will carry out flux quantization and obtain an explicit expression for the entropy. This will enable us to compare, successfully, with the general formalism of this paper in section 5.2.

The metric takes the form

$$
\mathrm{d} s^{2}\left(Y_{9}\right)=\frac{y^{3}-3 y+2 a}{y^{3}} D z^{2}+\frac{4 \mathrm{~d} y^{2}}{q(y)}+\frac{q(y)(D \psi)^{2}}{y^{3}\left(y^{3}-3 y+2 a\right)}+\frac{16}{y^{2}} \mathrm{~d} s^{2}\left(\mathrm{KE}_{6}^{+}\right),
$$

where $\mathrm{d} s^{2}\left(\mathrm{KE}_{6}^{+}\right)$is an arbitrary six-dimensional Kähler-Einstein metric, normalized so that $\rho_{\mathrm{KE}}=8 J_{\mathrm{KE}}$. Moreover, we have introduced the functions

$$
q(y)=y^{4}-4 y^{2}+4 a y-a^{2}, \quad g(y)=\frac{a-y}{y^{3}-3 y+2 a},
$$

and covariant derivatives

$$
D z \equiv \mathrm{d} z-g(y) D \psi, \quad D \psi \equiv \mathrm{d} \psi+4 B
$$

where $4 B$ is the natural connection on the canonical line bundle of $\mathrm{KE}_{6}^{+}$(i.e. $\mathrm{d} B=2 J_{\mathrm{KE}}$ ). The constant $a$, explicitly given in terms of $\mathrm{p}, \mathrm{q}$ below, lies in the range $0<a<1$. The quartic $q(y)$ then has four distinct roots, $y_{1}<y_{2}<y_{3}<y_{4}$, and we choose the range of $y$ to be $y_{2} \leq y \leq y_{3}$ where

$$
y_{2}=-1+\sqrt{1+a}, \quad y_{3}=1-\sqrt{1-a} .
$$

Potential conical singularities at $y=y_{2}, y_{3}$ are avoided by taking $\psi$ to be a periodic coordinate with period $2 \pi$. Finally, as explained in [18], the coordinate $z$ is periodic with period $2 \pi l$, with

$$
g\left(y_{3}\right)-g\left(y_{2}\right)=l \mathbf{q}, \quad g\left(y_{2}\right)=l \mathbf{p} / m
$$


where $\mathrm{p}, \mathrm{q}>0$ are relatively prime ${ }^{8}$ integers and the integer $m$ is the Fano index of $\mathrm{KE}_{6}^{+}$. These conditions are satisfied provided that

$$
\begin{aligned}
a & =\frac{m \mathrm{q}(2 \mathrm{p}+m \mathrm{q})}{2 \mathrm{p}^{2}+2 m \mathrm{pq}+m^{2} \mathrm{q}^{2}} \\
l & =\frac{m\left[\mathrm{p}^{2}+m \mathrm{pq}+\left(m^{2} / 2\right) \mathrm{q}^{2}\right]^{1 / 2}}{\mathrm{p}(\mathrm{p}+m \mathrm{q})}
\end{aligned}
$$

We now turn to flux quantization. We begin by noting that the two-form $F$, entering into the expression for the four-form $G$ in (2.15), is given by

$$
F=\frac{2^{3 / 2}}{3^{3 / 2}}\left(3 y^{2} \mathrm{~d} y \wedge \mathrm{d} z-8 a J_{\mathrm{KE}}\right) .
$$

After taking the $D=11$ Hodge dual of $G$ we obtain the seven-form given by

$$
\begin{aligned}
L^{-6} *_{11} G= & \frac{2^{14}}{3^{2}} \frac{q(y)}{y\left(y^{3}-3 y+2 a\right)} \frac{1}{3 !} J_{\mathrm{KE}}^{3} \wedge D \psi+\frac{2^{14}}{3^{2}} \frac{y-a}{y} \frac{1}{3 !} J_{\mathrm{KE}}^{3} \wedge D z \\
& +\frac{a}{y^{2}} \frac{2^{11}}{3^{3}} \frac{1}{2 !} J_{\mathrm{KE}}^{2} \wedge \mathrm{d} y \wedge D \psi \wedge D z .
\end{aligned}
$$

Flux quantization requires that

$$
\frac{1}{\left(2 \pi \ell_{p}\right)^{6}} \int_{\Sigma_{A}} *_{11} G=N_{A} \in \mathbb{Z}
$$

over all seven-cycles $\Sigma_{A} \subset Y_{9}$, where $A=1, \ldots$, rank $H_{7}\left(Y_{9}, \mathbb{Z}\right)$ runs over an integral basis for the free part of $H_{7}\left(Y_{9}, \mathbb{Z}\right)$, and $\ell_{p}$ denotes the eleven-dimensional Planck length.

To proceed further we need a basis for the free part of $H_{7}\left(Y_{9}, \mathbb{Z}\right)$. Recall that $Y_{9}$ is the total space of $\mathrm{U}(1)$ fibration, with fibre coordinate $z$, over an eight-dimensional manifold, $B_{8}$, the latter being the total space of an $S^{2}$ bundle over $\mathrm{KE}_{6}^{+}$. A basis for the free part of $H_{6}\left(B_{8}, \mathbb{Z}\right)$ is given by a section of the $S^{2}$ bundle over $\mathrm{KE}_{6}^{+}$, say at $y_{2}$ or $y_{3}$, together with the total spaces of the $S^{2}$ fibrations over each basis four-cycle $\Sigma_{a} \in H_{4}\left(\mathrm{KE}_{6}^{+}, \mathbb{Z}\right)$. It will be useful in a moment to write the Poincaré dual of the first Chern class of the $m$ th root of the canonical line bundle of $\mathrm{KE}_{6}^{+}$, denoted $c_{1}(\mathcal{N})$, as $\left[c_{1}(\mathcal{N})\right]=s_{a} \Sigma_{a}$, where $s_{a}$ are a set of co-prime integers.

Now, since the $\mathrm{U}(1)$ bundle over $B_{8}$ is non-trivial, all non-trivial seven-cycles come from the total space of the U(1) fibration over a six-cycle in $B_{8}$. Let us label these as follows: $D_{0}$ denotes the seven-cycle arising from the section $y=y_{2}, \tilde{D}_{0}$ is the cycle corresponding to $y=y_{3}$, and $D_{a}$ the cycle arising from $\Sigma_{a}$. Note that these cycles are not independent. From the $S^{2}$ fibration structure of $B_{8}$ we have

$$
D_{0}=\tilde{D}_{0}-m s_{a} D_{a}
$$

while the $\mathrm{U}(1)$ fibration is such that

$$
0=\mathrm{q} \tilde{D}_{0}+\mathrm{p} s_{a} D_{a} .
$$

\footnotetext{
${ }^{8}$ This condition ensures that $Y_{9}$ is simply connected.
} 
The flux integrals are then explicitly given by

$$
\begin{aligned}
& N\left(D_{0}\right)=-\frac{2^{2}}{3^{3} \pi^{2}}\left(\frac{L}{\ell_{p}}\right)^{6} \frac{m M}{\mathrm{p}}, \\
& N\left(\tilde{D}_{0}\right)=-\frac{2^{2}}{3^{3} \pi^{2}}\left(\frac{L}{\ell_{p}}\right)^{6} \frac{m M}{\mathrm{p}+m \mathrm{q}}, \\
& N\left(D_{a}\right)=\frac{2^{2}}{3^{3} \pi^{2}}\left(\frac{L}{\ell_{p}}\right)^{6} \frac{m^{4} \mathrm{q}}{\mathrm{p}(\mathrm{p}+m \mathrm{q})} n_{a},
\end{aligned}
$$

where

$$
M \equiv \int_{\mathrm{KE}_{6}^{+}}\left(\frac{\rho_{\mathrm{KE}}}{2 \pi}\right)^{3}=\int_{\mathrm{KE}_{6}^{+}} c_{1}^{3}
$$

and we have also used

$$
\int_{\Sigma_{a}}\left(\frac{\rho_{\mathrm{KE}}}{2 \pi}\right)^{2}=m^{2} n_{a}
$$

for some co-prime integers $n_{a} \equiv \int_{\Sigma_{a}} c_{1}(\mathcal{N})^{2}$, which follows from

$$
\frac{\rho_{\mathrm{KE}}}{2 \pi}=c_{1}=m c_{1}(\mathcal{N}),
$$

with $m^{3} s_{a} n_{a}=M$. Thus we should choose

$$
\left(\frac{L}{\ell_{p}}\right)^{6}=\frac{3^{3} \pi^{2}}{2^{2}} \frac{\mathrm{p}(\mathrm{p}+m \mathrm{q}) n}{h m^{4}}
$$

where $h=\operatorname{hcf}\left(M / m^{3}, \mathbf{q}\right)$ and $n$ is an integer, so that

$$
\begin{aligned}
& N\left(D_{0}\right)=-\frac{M}{h m^{3}}(\mathrm{p}+m \mathrm{q}) n, \\
& N\left(\tilde{D}_{0}\right)=-\frac{M}{h m^{3}} \mathrm{p} n, \\
& N\left(D_{a}\right)=\frac{\mathrm{q}}{h} n_{a} n
\end{aligned}
$$

are all integers. Notice these are consistent with the homology relations (D.12) and (D.13).

With these ingredients to hand, and using [18]

$$
\mathrm{e}^{B}=\frac{R}{2}=\left(\frac{3}{2}\right)^{3 / 2} \frac{1}{y^{3}}
$$

where $R$ is the Ricci scalar of the eight-dimensional transverse Kähler metric, we may now compute the "entropy" given by (2.17) (see equation (2.20) of [4])

$$
\begin{aligned}
\mathscr{S} & =\frac{1}{2^{6} \pi^{7}}\left(\frac{L}{\ell_{p}}\right)^{9} \int_{Y_{9}} \mathrm{e}^{-3 B} \mathrm{vol}_{9}, \\
& =\frac{2^{1 / 2}}{3^{1 / 2}} \frac{2^{6}}{3^{5} \pi^{2}}\left(\frac{L}{\ell_{p}}\right)^{9}\left(y_{3}-y_{2}\right) l M \\
& =\frac{2^{7 / 2} \pi M \sqrt{\mathrm{p}(m \mathrm{q}+\mathrm{p})}\left[\sqrt{(m \mathrm{q}+2 \mathrm{p})^{2}+m^{2} \mathrm{q}^{2}}-(m \mathrm{q}+2 \mathrm{p})\right]}{3 m^{5} h^{3 / 2}} n^{3 / 2} .
\end{aligned}
$$




\section{E Sasakian volume function}

As originally pointed out in [3], GK geometry shares many similarities with Sasakian geometry. In this appendix we point out that the formalism developed in $[9,16]$ and the present paper allows one to efficiently compute the Sasakian volume function of $[5,6]$ in many interesting cases.

Recall that a Sasakian manifold $\left(Y_{2 n+1}, \mathrm{~d} s_{2 n+1}^{2}\right)$ of real dimension $2 n+1$ with $n \geq 1$, may be defined as a Riemannian manifold whose metric cone (2.6) is Kähler. Precisely as for GK geometry in section 2, there is unit norm Killing vector $\xi$ on $Y_{2 n+1}$ with dual one-form $\eta$, so that the Sasakian metric may be written as

$$
\mathrm{d} s_{2 n+1}^{2}=\eta^{2}+\mathrm{d} s_{2 n}^{2}
$$

where $\mathrm{d} s_{2 n}^{2}$ is a Kähler metric transverse to the foliation $\mathcal{F}_{\xi}$ generated by $\xi$, cf. (2.2) for GK geometry. In Sasakian geometry $\eta$ is a contact one-form, satisfying $\mathrm{d} \eta=2 J$, where $J$ is the transverse Kähler form.

If one is interested in Sasaki-Einstein metrics, where the metric cone (2.6) is Ricci-flat Kähler, then there is necessarily a nowhere zero holomorphic $(n+1,0)$-form $\Psi$ on the cone satisfying

$$
\mathcal{L}_{\xi} \Psi=\mathrm{i}(n+1) \Psi
$$

Suppose that (E.2) holds on the Kähler cone. As in section 3.1 we may write the Reeb vector as

$$
\xi=\sum_{i=1}^{r+1} b_{i} \partial_{\varphi_{i}}
$$

where by definition $\Psi$ has unit charge under $\partial_{\varphi_{1}}$, and is uncharged under $\partial_{\varphi_{i}}, i=2,3, \ldots$, $r+1 \geq 1$. The condition (E.2) then implies that

$$
[\mathrm{d} \eta]=\frac{1}{b_{1}}[\rho] \in H_{B}^{2}\left(\mathcal{F}_{\xi}\right),
$$

precisely as in (3.6), except that for Sasakian geometry we should then set $b_{1}=n+1$ so that (E.2) holds.

If we now define the Sasakian volume

$$
\operatorname{Vol}\left(Y_{2 n+1}\right) \equiv \int_{Y_{2 n+1}} \eta \wedge \frac{J^{n}}{n !}
$$

where $\mathrm{d} \eta=2 J$ and (E.4) holds, then the above comments imply that

$$
\operatorname{Vol}\left(Y_{2+1}\right)=\frac{1}{2 n b_{1}} S_{\mathrm{SUSY}}\left(\xi,[J]=\frac{1}{2 b_{1}}[\rho]\right),
$$

where $S_{\text {SUSY }}$ is the supersymmetric action (2.10), and one should set $b_{1}=n+1$.

With the general formula (E.6) in hand, we may now compute the Sasakian volume function in the case that $Y_{2 n+1}$ is the total space of a toric $X_{2 r+1}$ fibration over a Kähler 
base $B_{2 k}$, where $n=r+k$, and with the Reeb vector $\xi$ tangent to the fibres. We present formulae for $k=1$ and $k=2$ below, together with some illustrative examples. We also note that the formulae are valid for Sasakian geometry on $Y_{2 n+1}$ with $n \geq 1$ (even though the analysis in the bulk of the paper was for GK geometry with $n \geq 3$ ).

Base $\boldsymbol{B}_{\mathbf{2}}$. Taking $k=1$, the base $B_{2}$ is a Riemann surface. However, in Sasakian geometry where (E.2) holds the Kähler class is a positive multiple of the anti-canonical class, which implies that $B_{2}=S^{2}$ necessarily has genus zero. In this case the formalism in section 4.2 (generalizing $[9,16]$ ) gives the general formula

$$
\operatorname{Vol}\left(Y_{2 n+1}\right)=-\left.\frac{1}{2 n b_{1}}\left[\frac{1}{b_{1}} \sum_{a=1}^{d} \frac{\partial \mathcal{V}}{\partial \lambda_{a}}+2 \pi b_{1} \sum_{i=1}^{r+1} n_{i} \frac{\partial \mathcal{V}}{\partial b_{i}}\right]\right|_{\lambda_{a}=-\frac{1}{2 b_{1}}, b_{1}=n+1},
$$

where $n=r+1$. Here as usual $\mathcal{V}$ denotes the master volume of the $X_{2 r+1}$ fibres, and we have defined

$$
n_{i} \equiv \frac{1}{2 \pi} \int_{S^{2}} F_{i}
$$

which describes the twisting of the fibres over the base $B_{2}=S^{2}$. As for GK geometry, the existence of a holomorphic $(n+1,0)$-form $\Psi$ on the metric cone over $Y_{2 n+1}$ implies that $n_{1}=2$, which is the anti-canonical class of $S^{2}$ in $\mathbb{Z} \cong H^{2}\left(S^{2}, \mathbb{Z}\right)$. On the right hand side of (E.7) one should set all $\lambda_{a}=-\frac{1}{2 b_{1}}, a=1, \ldots, d$, after taking derivatives with respect to the $\lambda_{a}$, and also $b_{1}=n+1$, after taking derivatives with respect to the $b_{i}$. The former condition ensures that the Kähler class of the fibres satisfies $[\omega]=\frac{1}{2 b_{1}}[\rho]$, as in (3.14).

To illustrate (E.7), let us consider the case of three-dimensional fibres, with $r=1$. In this case $Y_{5}$ is the total space of a Lens space $X_{3} \cong S^{3} / \mathbb{Z}_{p}$ fibration over $S^{2}$, where the master volume $\mathcal{V}$ of the fibres is given by (B.6). Using (E.7) we easily compute the Sasakian volume

$$
\operatorname{Vol}\left(Y_{5}\right)=\frac{p\left[2 b_{2}\left(p-n_{2}\right)+3 p n_{2}\right]}{b_{2}^{2}\left(b_{2}-3 p\right)^{2}} \pi^{3}
$$

Setting the twisting variable $n_{2}=p+q$, with ${ }^{9} p>q>0$, extremizing (E.9) over $b_{2}$ one finds that the unique critical point inside the Reeb cone is

$$
b_{2}=\frac{p\left(2 p+3 q-\sqrt{4 p^{2}-3 q^{2}}\right)}{2 q} .
$$

\footnotetext{
${ }^{9}$ The inequality comes from requiring the metric cone over $Y_{5}$ to be an affine variety. This is perhaps easiest to see using toric geometry, since $Y_{5}$ is here toric. In terms of the fibration picture described in the present paper, recall that $X_{3} \cong S^{3} / \mathbb{Z}_{p}$ is the link of the $A_{p-1}$ singularity $C\left(X_{3}\right)=\mathbb{C}^{2} / \mathbb{Z}_{p}$. The corresponding $A_{p-1}$ fibration over $S^{2}$ is then a partial resolution of the affine cone $C\left(Y_{5}\right)$. The two divisors $z_{1}=0$ and $z_{2}=0$ in the fibre $\mathbb{C}^{2} / \mathbb{Z}_{p}$ give rise to $\mathbb{C} / \mathbb{Z}_{p}$ fibrations over $S^{2}$, with Chern numbers $-n_{2}=-(p+q)$ and $-2 p+n_{2}=-(p-q)$. Both should be negative, in order that the total spaces are holomorphically convex. For more general examples there will be similar convexity conditions on the twisting parameters that need to be imposed.
} 
The on-shell volume is then

$$
\operatorname{Vol}\left(Y_{5}\right)=\frac{q^{2}\left(2 p+\sqrt{4 p^{2}-3 q^{2}}\right)}{3 p^{2}\left(-2 p^{2}+3 q^{2}+p \sqrt{4 p^{2}-3 q^{2}}\right)} \pi^{3},
$$

which agrees with the volume of the $Y^{p, q}$ Sasaki-Einstein manifolds [22], as expected.

Base $\boldsymbol{B}_{4}$. Taking $k=2$, the base $B_{4}$ is now a Kähler surface. Again, this should be Fano, having positive anti-canonical class. In this case we find

$$
\begin{aligned}
\operatorname{Vol}\left(Y_{2 n+1}\right)= & -\frac{1}{2 n b_{1}}\left[\frac{\pi^{2}}{2 b_{1}^{2}} \sum_{a=1}^{d} \frac{\partial \mathcal{V}}{\partial \lambda_{a}} \int_{B_{4}} c_{1}^{2}+\pi \sum_{i=1}^{r+1} \frac{\partial \mathcal{V}}{\partial b_{i}} \int_{B_{4}} F_{i} \wedge c_{1}\right. \\
& \left.-\frac{b_{1}}{r+1} \sum_{a=1}^{d} \sum_{i, j=1}^{r+1} \lambda_{a} \frac{\partial^{2} \mathcal{V}}{\partial b_{j} \partial v_{a}^{i}} \int_{B_{4}} \frac{1}{2} F_{i} \wedge F_{j}\right]\left.\right|_{\lambda_{a}=-\frac{1}{2 b_{1}}, b_{1}=n+1},
\end{aligned}
$$

where now $n=r+2$. As in GK geometry we have $\left[F_{1}\right]=2 \pi c_{1} \in H^{2}\left(B_{4}, \mathbb{R}\right)$, where $c_{1}=c_{1}\left(B_{4}\right)$ is the anti-canonical class of the base $B_{4}$.

Again, we illustrate (E.12) by taking three-dimensional fibres, with $r=1$, and choose the base to be $B_{4}=\mathbb{C} P^{2}$. In this case there is a single generator of the cohomology $H=1 \in \mathbb{Z} \cong H^{2}\left(\mathbb{C} P^{2}, \mathbb{Z}\right)$, given by the hyperplane class, with $\int_{\mathbb{C} P^{2}} H^{2}=1$. Writing $\left[F_{2}\right]=2 \pi \kappa H$ with $\kappa \in \mathbb{Z}$, and using $c_{1}=3 H$, we compute

$$
\operatorname{Vol}\left(Y_{7}\right)=\frac{p\left[\kappa^{2}\left(16 p^{2}-12 b_{2} p+3 b_{2}^{2}\right)+3 b_{2} \kappa p\left(4 p-3 b_{2}\right)+9 p^{2} b_{2}^{2}\right]}{3 b_{2}^{3}\left(4 p-b_{2}\right)^{3}} \pi^{4} .
$$

Extremizing over $b_{2}$, we find that the on-shell volume agrees with the $Y^{p, \kappa}\left(\mathbb{C} P^{2}\right)$ SasakiEinstein manifolds of [23], ${ }^{10}$ as expected.

Open Access. This article is distributed under the terms of the Creative Commons Attribution License (CC-BY 4.0), which permits any use, distribution and reproduction in any medium, provided the original author(s) and source are credited.

\section{References}

[1] N. Kim, $A d S_{3}$ solutions of IIB supergravity from D3-branes, JHEP 01 (2006) 094 [hep-th/0511029] [INSPIRE].

[2] N. Kim and J.-D. Park, Comments on AdS $S_{2}$ solutions of $D=11$ supergravity, JHEP 09 (2006) 041 [hep-th/0607093] [INSPIRE].

[3] J.P. Gauntlett and N. Kim, Geometries with Killing Spinors and Supersymmetric AdS Solutions, Commun. Math. Phys. 284 (2008) 897 [arXiv:0710.2590] [INSPIRE].

\footnotetext{
${ }^{10}$ See also [24], where the $Y^{p, \kappa}\left(\mathbb{C} P^{2}\right)$ notation was more precisely defined. We note that there is a typographical error in the volume in equation (2.23) of that paper, where the right hand side should be multiplied by $\frac{1}{4}$ to obtain the Sasaki-Einstein volume with standard normalization we are using in this appendix.
} 
[4] C. Couzens, J.P. Gauntlett, D. Martelli and J. Sparks, A geometric dual of c-extremization, JHEP 01 (2019) 212 [arXiv:1810.11026] [INSPIRE].

[5] D. Martelli, J. Sparks and S.-T. Yau, The Geometric dual of a-maximisation for Toric Sasaki-Einstein manifolds, Commun. Math. Phys. 268 (2006) 39 [hep-th/0503183] [INSPIRE].

[6] D. Martelli, J. Sparks and S.-T. Yau, Sasaki-Einstein manifolds and volume minimisation, Commun. Math. Phys. 280 (2008) 611 [hep-th/0603021] [INSPIRE].

[7] F. Benini and N. Bobev, Exact two-dimensional superconformal R-symmetry and c-extremization, Phys. Rev. Lett. 110 (2013) 061601 [arXiv:1211.4030] [INSPIRE].

[8] F. Benini, K. Hristov and A. Zaffaroni, Black hole microstates in AdS $S_{4}$ from supersymmetric localization, JHEP 05 (2016) 054 [arXiv:1511.04085] [INSPIRE].

[9] J.P. Gauntlett, D. Martelli and J. Sparks, Toric geometry and the dual of I-extremization, JHEP 06 (2019) 140 [arXiv: 1904.04282] [INSPIRE].

[10] S.M. Hosseini and A. Zaffaroni, Geometry of $\mathcal{I}$-extremization and black holes microstates, JHEP 07 (2019) 174 [arXiv: 1904.04269] [INSPIRE].

[11] F. Benini, K. Hristov and A. Zaffaroni, Exact microstate counting for dyonic black holes in $A d S_{4}$, Phys. Lett. B 771 (2017) 462 [arXiv: 1608.07294] [INSPIRE].

[12] A. Cabo-Bizet, V.I. Giraldo-Rivera and L.A. Pando Zayas, Microstate counting of $A d S_{4}$ hyperbolic black hole entropy via the topologically twisted index, JHEP 08 (2017) 023 [arXiv: 1701.07893] [INSPIRE].

[13] F. Azzurli, N. Bobev, P.M. Crichigno, V.S. Min and A. Zaffaroni, A universal counting of

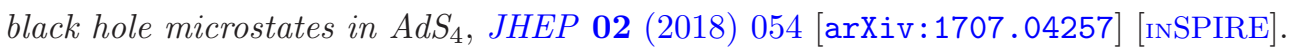

[14] F. Benini, H. Khachatryan and P. Milan, Black hole entropy in massive Type IIA, Class. Quant. Grav. 35 (2018) 035004 [arXiv:1707.06886] [INSPIRE].

[15] S.M. Hosseini, K. Hristov and A. Passias, Holographic microstate counting for AdS $S_{4}$ black holes in massive IIA supergravity, JHEP 10 (2017) 190 [arXiv:1707.06884] [INSPIRE].

[16] J.P. Gauntlett, D. Martelli and J. Sparks, Toric geometry and the dual of c-extremization, JHEP 01 (2019) 204 [arXiv: 1812.05597] [INSPIRE].

[17] J.P. Gauntlett, O.A.P. Mac Conamhna, T. Mateos and D. Waldram, Supersymmetric AdS $S_{3}$ solutions of type IIB supergravity, Phys. Rev. Lett. 97 (2006) 171601 [hep-th/0606221] [INSPIRE].

[18] J.P. Gauntlett, N. Kim and D. Waldram, Supersymmetric $A d S_{3}, A d S_{2}$ and Bubble Solutions, JHEP 04 (2007) 005 [hep-th/0612253] [INSPIRE].

[19] S.M. Hosseini and A. Zaffaroni, Proving the equivalence of c-extremization and its gravitational dual for all toric quivers, JHEP 03 (2019) 108 [arXiv:1901.05977] [INSPIRE].

[20] J.P. Gauntlett and O.A.P. Mac Conamhna, AdS spacetimes from wrapped D3-branes, Class. Quant. Grav. 24 (2007) 6267 [arXiv:0707.3105] [INSPIRE].

[21] O.A.P. Mac Conamhna and E. O Colgain, Supersymmetric wrapped membranes, AdS $S_{2}$ spaces and bubbling geometries, JHEP 03 (2007) 115 [hep-th/0612196] [INSPIRE]. 
[22] J.P. Gauntlett, D. Martelli, J. Sparks and D. Waldram, Sasaki-Einstein metrics on $S^{2} \times S^{3}$, Adv. Theor. Math. Phys. 8 (2004) 711 [hep-th/0403002] [InSPIRE].

[23] J.P. Gauntlett, D. Martelli, J.F. Sparks and D. Waldram, A New infinite class of Sasaki-Einstein manifolds, Adv. Theor. Math. Phys. 8 (2004) 987 [hep-th/0403038] [INSPIRE].

[24] D. Martelli and J. Sparks, Notes on toric Sasaki-Einstein seven-manifolds and $A d S_{4} / C F T_{3}$, JHEP 11 (2008) 016 [arXiv:0808.0904] [INSPIRE]. 